\title{
Effects of oxidation on tensile deformation of iron nanowires: Insights from reactive molecular dynamics simulations
}

Gurcan Aral', Yun-Jiang Wang, Shigenobu Ogata, and Adri C. T. van Duin

Citation: Journal of Applied Physics 120, 135104 (2016); doi: 10.1063/1.4963828

View online: http://dx.doi.org/10.1063/1.4963828

View Table of Contents: http://aip.scitation.org/toc/jap/120/13

Published by the American Institute of Physics

\section{Articles you may be interested in}

Effect of native oxide layers on copper thin-film tensile properties: A reactive molecular dynamics study Journal of Applied Physics 118, 235306235306 (2015); 10.1063/1.4938384

A reactive molecular dynamics simulation of the silica-water interface

Journal of Applied Physics 132, 174704174704 (2010); 10.1063/1.3407433

Properties of high-density, well-ordered, and high-energy metallic glass phase designed by pressurized quenching

Journal of Applied Physics 109, 091906091906 (2016); 10.1063/1.4962128

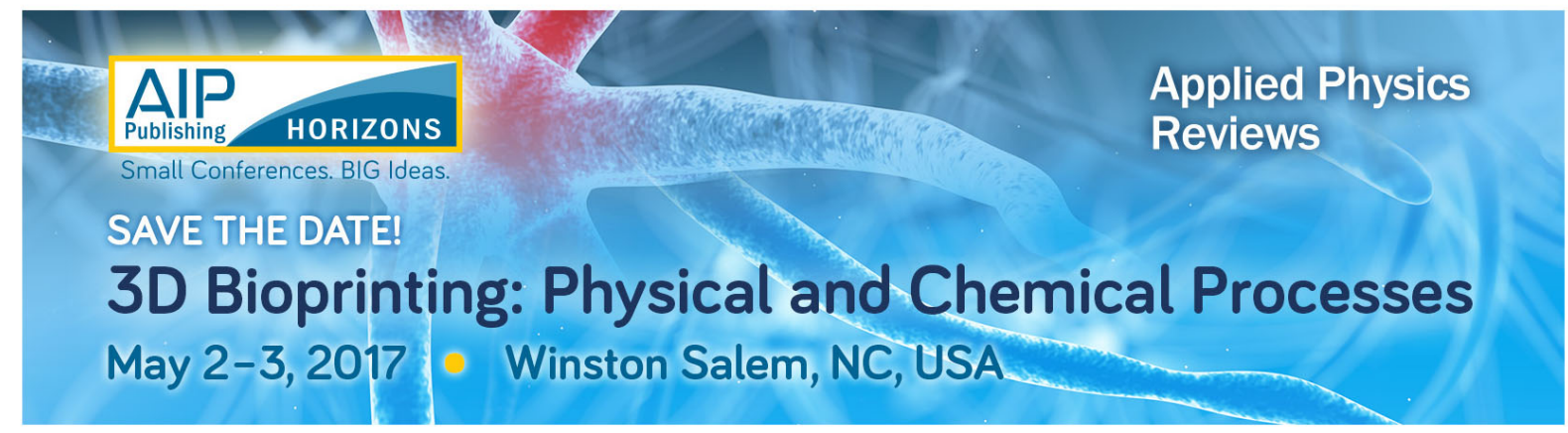




\title{
Effects of oxidation on tensile deformation of iron nanowires: Insights from reactive molecular dynamics simulations
}

\author{
Gurcan Aral, ${ }^{1, a)}$ Yun-Jiang Wang, ${ }^{2}$ Shigenobu Ogata, ${ }^{3,4}$ and Adri C. T. van Duin ${ }^{5}$ \\ ${ }^{1}$ Department of Physics, Izmir Institute of Technology, Urla, Izmir 35430, Turkey \\ ${ }^{2}$ State Key Laboratory of Nonlinear Mechanics, Institute of Mechanics, Chinese Academy of Sciences, \\ Beijing 100190, China \\ ${ }^{3}$ Department of Mechanical Science and Bioengineering, Graduate School of Engineering Science, \\ Osaka University, Osaka 560-8531, Japan \\ ${ }^{4}$ Center for Elements Strategy Initiative for Structural Materials (ESISM), Kyoto University, Kyoto 606-8501, Japan \\ ${ }^{5}$ Department of Mechanical and Nuclear Engineering, The Pennsylvania State University, University Park, \\ Pennsylvania 16802, USA
}

(Received 21 May 2016; accepted 18 September 2016; published online 4 October 2016)

\begin{abstract}
The influence of oxidation on the mechanical properties of nanostructured metals is rarely explored and remains poorly understood. To address this knowledge gap, in this work, we systematically investigate the mechanical properties and changes in the metallic iron $(\mathrm{Fe})$ nanowires (NWs) under various atmospheric conditions of ambient dry $\mathrm{O}_{2}$ and in a vacuum. More specifically, we focus on the effect of oxide shell layer thickness over Fe NW surfaces at room temperature. We use molecular dynamics (MD) simulations with the variable charge ReaxFF force field potential model that dynamically handles charge variation among atoms as well as breaking and forming of the chemical bonds associated with the oxidation reaction. The ReaxFF potential model allows us to study large length scale mechanical atomistic deformation processes under the tensile strain deformation process, coupled with quantum mechanically accurate descriptions of chemical reactions. To study the influence of an oxide layer, three oxide shell layer thicknesses of $\sim 4.81 \AA$, $\sim 5.33 \AA$, and $\sim 6.57 \AA$ are formed on the pure Fe NW free surfaces. It is observed that the increase in the oxide layer thickness on the Fe NW surface reduces both the yield stress and the critical strain. We further note that the tensile mechanical deformation behaviors of Fe NWs are dependent on the presence of surface oxidation, which lowers the onset of plastic deformation. Our MD simulations show that twinning is of significant importance in the mechanical behavior of the pure and oxide-coated Fe NWs; however, twin nucleation occurs at a lower strain level when Fe NWs are coated with thicker oxide layers. The increase in the oxide shell layer thickness also reduces the external stress required to initiate plastic deformation. Published by AIP Publishing.
\end{abstract}

[http://dx.doi.org/10.1063/1.4963828]

\section{INTRODUCTION}

Owing to their unique physical, chemical, and mechanical properties, the chemically reactive iron element ${ }^{1,2}$ and its oxide nanostructure compounds are widely used as most promising materials for diverse nanoscale technological applications, such as heterogeneous photo catalysts/catalysis, ${ }^{3,4}$ magnetic recording media, ${ }^{5-7}$ hydrogen storage, ${ }^{4}$ field emission devices, ${ }^{8}$ water splitting, ${ }^{8,9}$ chemical, gas, and bio sensors ${ }^{6,10-12}$ permanent magnets, ${ }^{6}$ biomedical applications, ${ }^{5,13,14}$ and solar cells. ${ }^{9,11}$ In ambient environment conditions, even at room temperature, spontaneous oxidations of metallic Fe nanowires (NWs) exhibit rather complex phenomena when in contact with oxidizing agents, such as oxygen and/or water. ${ }^{1,2}$ Thus, the segregation and reconstruction processes at the free NW surface resulting from chemisorption of oxygen increase the surface-to-volume ratio, which is substantial at the nanometer scale, allowing a thin oxide surface layer with multiple phases to be formed. ${ }^{15}$ While iron oxides with many phases are commonly found in nature, of

\footnotetext{
a) Author to whom correspondence should be addressed. Electronic mail: gurcanaral@iyte.edu.tr.
}

particular interest here are wüstite $(\mathrm{FeO})$, magnetite $\left(\mathrm{Fe}_{3} \mathrm{O}_{4}\right)$, and hematite $\left(\alpha-\mathrm{Fe}_{2} \mathrm{O}_{3}\right){ }^{1,2,15-17}$

Surface oxide effects become particularly significant at the nanometer scale, where even subtle variations in the oxide layer thickness and shape have major effects on their physical, chemical, and mechanical properties as well as their functionalities and performances for numerous applications, magnetic applications in particular. ${ }^{7}$ Recently, coating and synthesis of metallic Fe NWs with oxidizing agents have been attempted by many researchers in order to functionalize them or improve their wear and corrosion resistance for practical applications. ${ }^{17}$ In the past decade, several advanced experimental growth techniques have been proposed, such as vapor-solid growth, ${ }^{18}$ micro emulsion, ${ }^{10}$ thermal oxidation, ${ }^{12,19}$ and chemical vapor deposition, ${ }^{8}$ and have been successfully applied for the synthesis of $\mathrm{Fe}-\mathrm{Fe}_{\mathrm{x}} \mathrm{O}_{\mathrm{y}}$ nano composite materials. The required well-defined structure, a uniform density, a prescribed diameter, and a morphology with unique size and shape of the $\mathrm{Fe}_{\mathrm{x}} \mathrm{O}_{\mathrm{y}}$ shell layer around Fe NWs, which directly play a decisive role in the performance of $\mathrm{Fe}-\mathrm{Fe}_{\mathrm{x}} \mathrm{O}_{\mathrm{y}}$ composite materials, are difficult to control accurately. ${ }^{7,12}$ Therefore, to obtain an atomic-level 
comprehensive understanding of factors influencing the design and characteristics of the $\mathrm{Fe}-\mathrm{Fe}_{\mathrm{x}} \mathrm{O}_{\mathrm{y}}$ interfaces, oxidation of metallic $\mathrm{Fe}$ and the resulting oxide structure have been extensively studied theoretically for various experimental conditions. ${ }^{1,2,13,14}$ Oxidation of Fe single-crystal surfaces with three different orientations, along with the developed oxide microstructure on the free reactive surface, was studied by Subbaraman and colleagues as a function of temperature and $\mathrm{O}_{2}$ molecules' pressure. ${ }^{1}$ Their results reveal that the kinetics of oxidation and the growth rate of the oxide layer follow a logarithmic time dependence and are also affected by the surface orientations, temperature, and pressure, with thicknesses ranging from 1 to $2 \mathrm{~nm}$. Jeon et al. investigated the oxidation of nano crystalline $\mathrm{Fe}$ (100), (110), and (111) surface orientations using the ReaxFF for various temperatures with or without an external electric field. ${ }^{2}$ These authors have shown that the chemical composition of the oxide films formed at the free surface depends strongly on the applied external field.

In extant research, mechanical deformation and surface oxidation mechanisms of Fe NWs are typically studied separately. However, it is widely accepted that the surface oxide layer has a significant effect on the mechanical behaviors of metallic NWs. ${ }^{20,21}$ In particular, it remains difficult to quantify the effect of the oxide independently from the tensile mechanical deformation behavior and the Fe NW properties. More recently, both experimental and theoretical investigations specifically indicated that the oxide-coated NWs exhibit different mechanical properties than their pure (unoxidized) counterparts. For example, oxide-coated tungsten NWs exhibit increased Young's modulus, ${ }^{22}$ whereas it decreases in oxide-covered silicon ${ }^{23}$ and copper NWs. ${ }^{24}$ Sen et al. have also studied oxide shell layer dependent deformation mechanisms of metallic aluminum NWs by using the ReaxFF model. ${ }^{21,22}$ The authors observed that the oxide amorphous shell layer on the $\mathrm{Al} \mathrm{NW}$ free surface enhances dislocation processes, as well as the $\mathrm{Al}$ core's dislocation nucleation stress. Most importantly, the presence of native amorphous oxide shell layers on Al NWs was shown to play a significant role in the change in the elastic mechanical response from brittle to ductile transition behavior. In their recent work, Landau et al. presented mechanical behavior, deformation mechanism, and mechanical properties of the as-fabricated and helium-implanted single crystalline iron nano pillars through both tensile and compression experimental tests. ${ }^{25}$ The authors reported that, during a compressive deformation, both the as-fabricated and heliumimplanted samples display three distinct regimes: elastic loading, substantial strain hardening, and a "steady state" region. The authors also revealed that the presence of helium in the $\mathrm{Fe}$ nano pillars introduced significant changes to the intrinsic mechanical properties of Fe NWs. For example, yield and ultimate strength, as well as flow stress, significantly increase for helium-implanted samples compared to the as-fabricated ones. In a recent comprehensive molecular dynamics (MD) study by Sainath et al., the mechanical deformation properties and related atomistic mechanisms of metallic Fe nanowires were investigated as a function of various experimental conditions by using embedded-atom method (EAM) interatomic potentials. ${ }^{6,26,27}$ In their first study, ${ }^{26}$ the authors indicated that the deformation mode was dependent on the loading direction for $\langle 110\rangle /\{111\}$-orientated metallic Fe NWs. Mainly, two different deformation mechanisms were observed: twinning in tension and full dislocation slip in compressive loading. In their subsequent work, ${ }^{6}$ the authors studied the effect of the size-dependent tensile deformation mechanism for the $\langle 100\rangle /\{100\}$-orientated Fe NWs with the cross section wide in the range of $11.42-24.27 \mathrm{~nm}$. They reported that these NWs were deformed by the dislocation slip mechanism irrespective of the nanowire size. Finally, ${ }^{27}$ the same research group investigated the effect of Fe NW orientation on the mechanical deformation mechanisms with various initial orientations. The authors noted that the plastic deformation mechanism by twinning on the $\langle 111\rangle /\{112\}$ slip system was exhibited for $\langle 100\rangle,\langle 112\rangle$, and $\langle 102\rangle$ initial orientations only, whereas deformation observed in $\langle 110\rangle$ and $\langle 111\rangle$-oriented NWs was markedly different from that noted in crystal structures with other orientations, which exhibited a dislocation slip plastic mode. Hepburn and Ackland developed an EAM potential for $\mathrm{Fe}$ to investigate the dislocation-related properties and phenomena, to simulate the tension-compression asymmetry in nano pillars of bcc Fe. ${ }^{28,29}$ They showed that twinning occurs through the creation and expansion of twin boundaries for the $\langle 100\rangle /\{110\}$-orientated body center cubic (bcc) Fe NWs. The major deformation mechanism in the compressive case was the dislocation slip, observed during the plastic deformation process, suggesting that compressive and tension conditions are characterized by different plastic deformation mechanisms. ${ }^{29}$ The uniaxial deformation mechanisms of cylindrical [001]-oriented $\mathrm{Fe}$ and $\mathrm{FeCr}$ NWs were studied by using both EAM and Tersoff-like bond order potentials. ${ }^{30}$ Both potentials reveal that twinning is the preferred plastic deformation mechanism over full slip before necking. The results obtained suggest that, when the NWs are subjected to strain, deformation twinning initially occurs as $\langle 111\rangle /\{211]$ stacking fault and, as a result, the initial axial direction [100] of NWs transforms into a [011]-oriented wire over the twin boundary. According to Li et al., Mo, W, and Fe bcc NWs deform super elastically, where the $\langle 111\rangle /\{112\}$ dislocation slip is primarily responsible for the twinning/ detwinning mechanism that involves minimization of surface energy and twin boundary motion. ${ }^{31}$ Moreover, to provide a more detailed description of twin formation, in contrast to the metallic face center cubic (fcc) NWs, the authors noted that the bcc metallic NWs have a higher energy barrier for the nucleation of a twin but a lower barrier for twin formation and migration. Twinning has been reported in many simulations of Fe NWs. Nonetheless, the twinning deformation in bec metallic NWs is believed to occur experimentally at low temperatures or/and at high-strain rates. ${ }^{25}$ However, more recently, using high-resolution transmission electron microscopy, deformation twinning has been observed experimentally for bcc tungsten NWs. ${ }^{32}$ In spite of their importance, oxide shell layer thickness-related size effects for metallic NWs deformed by tensile force remain elusive and no systematic connection has been established to the native oxide layer effects on the mechanical deformation 
mechanism and properties of Fe NWs. For this reason, the correlations and effects of the oxide layer on the mechanical deformation performance must be investigated systematically to obtain a comprehensive understanding of the complex mechanical deformation processes occurring in Fe NWs as a function of the oxide shell layer thickness, as well as to determine the elastic mechanical properties of Fe NWs with and without surface oxide defect under tensile loading.

In this paper, the tensile deformation process and related material properties of Fe NWs with and without the oxide shell layer are presented and the findings compared to experimental and theoretical data. We have carried out MD simulation using the ReaxFF potential model proposed by Aryanpour $e t$ al. ${ }^{33}$ The remainder of the paper is organized as follows: In Section II, the effective reactive interaction potential model is described, while the detailed description of the simulation method and the computational schedule is given in Section III. The role of oxidation in the tensile mechanical deformation process and the effect of oxide shell layers on stress-strain behavior are discussed in Section IV. Moreover, the kinetic properties of oxidation and the microstructure of the oxide shell layer are analyzed by using pair correlation functions, coordination numbers, and bond angle distributions, and the elastic mechanical properties are also studied. Conclusions and final remarks are given in Section V.

\section{COMPUTATIONAL APPROACH AND METHODS}

Variable charge methods allow studying oxidation of metals ${ }^{34-36}$ metal alloys, ${ }^{37}$ dissimilar metal/metal oxide interfaces,${ }^{37}$ combustion chemistry, ${ }^{34}$ etc. For example, one of the variable-charge potential schemes was proposed by Streitz and Mintmire to treat the atomic charges as dynamic variables and was successfully applied to explore the oxidation mechanism of metallic $\mathrm{Al}$ nano systems as a function of temperature, oxygen pressure, and orientation of the aluminum substrate. ${ }^{33-36,38}$ Our MD simulations were carried out using the variable-charge scheme, known as the ReaxFF model, which can simulate the temporal evolution of atomic charges resulting from changes in the local environment with significantly lower amount of computational cost than is required when the quantum-based computational methods are employed. ${ }^{39}$ The most important feature of using the ReaxFF model to describe both covalent and (partially) ionic bonds, as well as the entire range of intermediate interactions, is that it can accurately describe mechanically induced chemical reactions by mechanical stretching, which is characterized by bond straightening, bond breakage, bond formation, inner atomic distortion, and rupture for pure and oxidized Fe NW systems. ${ }^{39}$ The ReaxFF potential, using a classical MD simulation method, is implemented in a parallel version of LAMMPS $^{40}$ that can be employed for systematic and detailed studies of complex intrinsic defect nucleation mechanisms and deformation processes occurring in Fe NWs with correlation of surface oxide. This ReaxFF model was successfully applied to study a wide range of oxides, such as $\mathrm{Si}^{41} \mathrm{Ni}^{42}{ }^{42} \mathrm{Ti}^{43} \mathrm{Pt}^{44}$ and $\mathrm{Al}$ oxides. ${ }^{2}$ Electrostatic potential energy is the function of the position vector of atoms as well as ionic charges; therefore, the chargedependent electrostatic force acts on the atoms, forcing them to exchange their respective valence charges. ${ }^{45}$ The ReaxFF variable-charge approach dynamically determines atomic charges $\left\{\mathrm{q}_{\mathrm{i}} \mid \mathrm{i}=1, \ldots, \mathrm{N}\right\}$ at each MD step by applying an electronegativity equalization method in order to minimize the electrostatic and polarization potential energy, with the charge-neutrality constraint, $\Sigma_{\mathrm{i}} \mathrm{q}_{\mathrm{i}}=0 .{ }^{45}$

\section{SYSTEM SETUP FOR MECHANICANOCHEMICAL DEFORMATION SIMULATIONS}

The initial configuration for the pure and oxide-coated cylindrical Fe NWs is schematically shown in Figs. 1(a) and 1(b) and Figs. 1(f) and 1(g), where blue and red colors represent $\mathrm{O}$ and $\mathrm{Fe}$ atoms, respectively. The Fe lattice has a bcc structure, with a lattice constant $a=2.86 \AA$. An initially pure solid cylindrical Fe NW is comprised of 24050 iron atoms with a $5.0 \mathrm{~nm}$ diameter and a lateral size of $14.3 \mathrm{~nm}$ length in the $z$ direction, where the lateral direction corresponds to the tensile direction. The diameter-to-length aspect ratio of the pure Fe NW is $\sim 1: 3$. The pure Fe NW was placed in the middle of an MD box oriented in the [001] direction, before being exposed to molecular oxygen. More specifically, 2000 $\mathrm{O}_{2}$ molecules in the dry gas phase were placed randomly into the region above the cylindrical Fe NW surface. To create the three different oxide shell layer thicknesses on the free Fe NW surface, all oxygen molecules were totally removed from the gas environment when an corresponding oxide shell layer thickness forms $\sim 4.81 \AA, \sim 5.33 \AA$, and $\sim 6.57 \AA$, in order to prevent further oxidation of Fe NW so as to keep the oxide layer thickness constant. The oxidation simulation with applying periodic boundary condition all direction was performed in the canonical ensemble (NVT), whereby heat bath was applied to all atoms, which was kept at a constant temperature of $\mathrm{T}=300 \mathrm{~K}$ during using NoseHoover thermostat chain. ${ }^{46}$ This approach prevented the ejection of $\mathrm{Fe}$ atoms from the surface. Consequently, they exhibited a typical core-shell $\left(\mathrm{Fe}-\mathrm{Fe}_{\mathrm{x}} \mathrm{O}_{\mathrm{y}}\right)$ structure, as illustrated in Figs. 1(f) and 1(g). The ratio of the number of Fe atoms to that of $\mathrm{O}$ atoms would affect the overall oxide thickness, surface morphology, and microstructures at each Fe NW surface. Series of snapshots are presented in Figs. $1(\mathrm{c})-1$ (f) to illustrate the oxidation process, and a view of the resulting oxide Fe NW geometry with rough surface is shown from different perspectives in Figs. 1(f) and 1(g). After oxidation simulation, periodic boundary conditions are just applied in the axial direction (tensile loading direction) to eliminate surface effects, with free boundary conditions along the other axes.

For preparation of mechanical testing, starting from the initial construction of all Fe NW configurations, energy minimization was first performed for all NWs by using conjugate gradient methods. During relaxation processes, all NWs were relaxed and equilibrated with a Nose/Hoover isobaricisothermal (NPT) at a constant temperature of $\mathrm{T}=300 \mathrm{~K}$, to allow structural relaxation to remove surface tension upon construction and internal stresses. ${ }^{47}$ On the other hand, all mechanical tensile MD simulations were performed in the NVT ensemble at a constant temperature of $\mathrm{T}=300 \mathrm{~K}$ using a Nose-Hoover thermostat. The velocity-Verlet algorithm was 
(a)

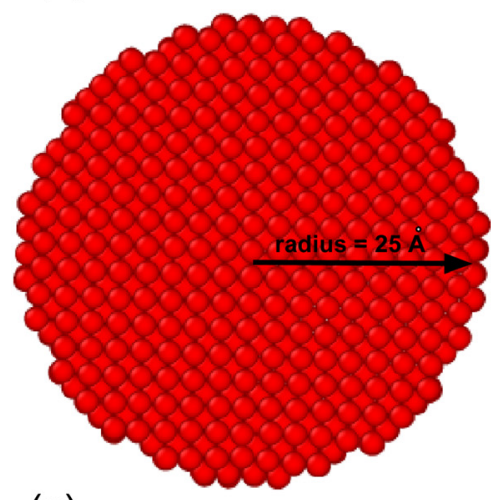

(c)
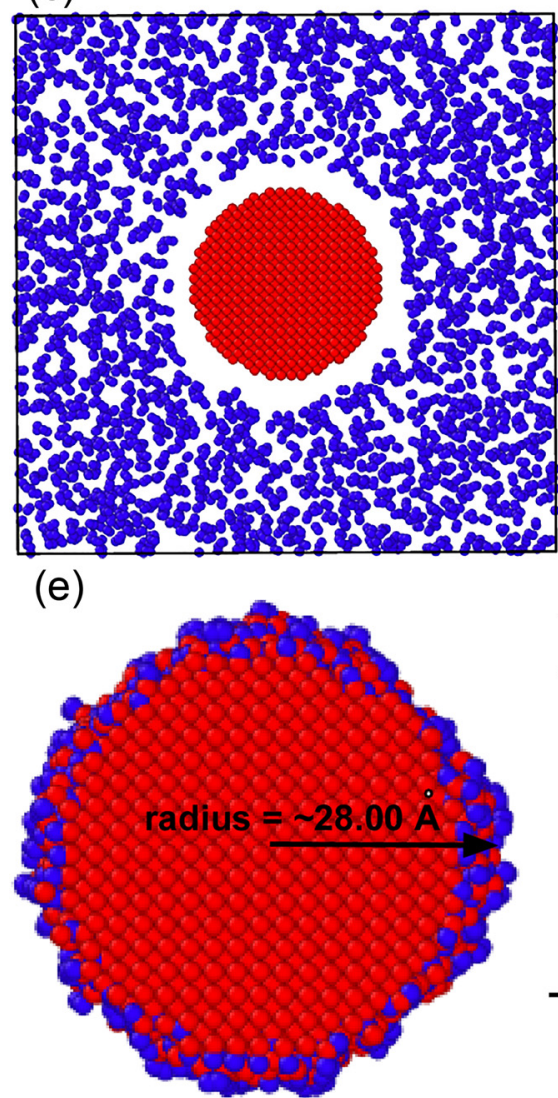

(b)

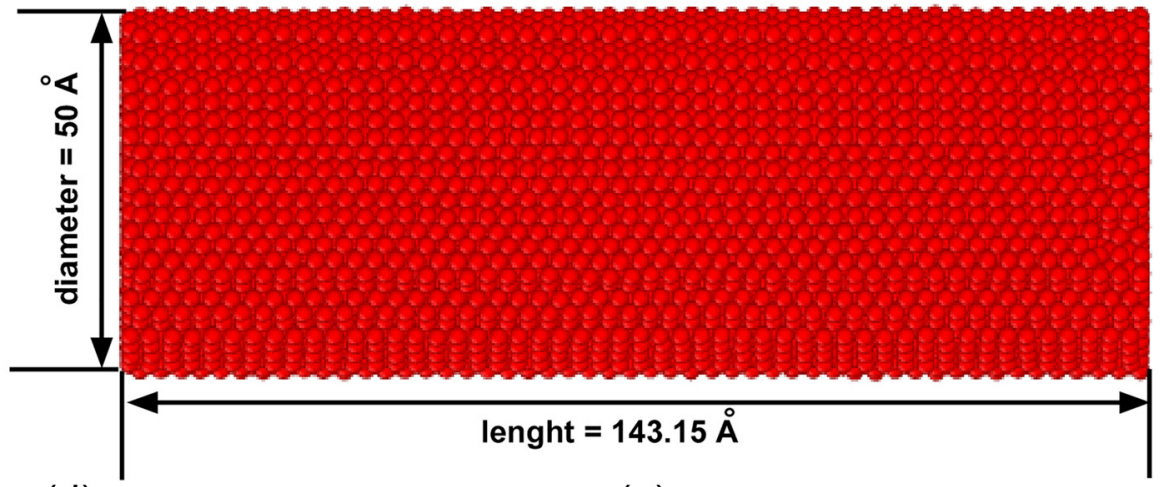

(d)

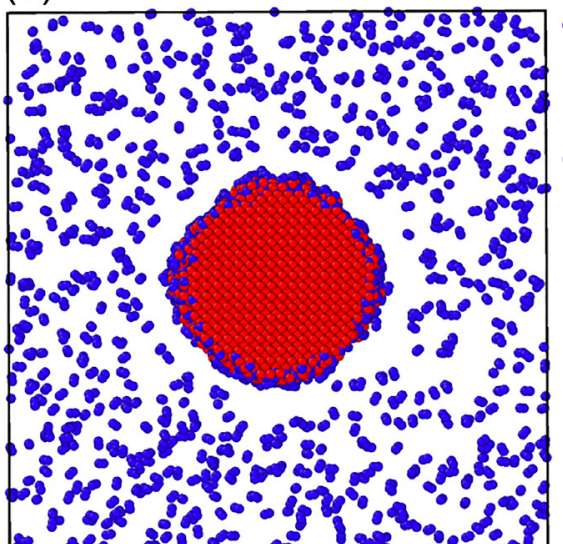

(e)
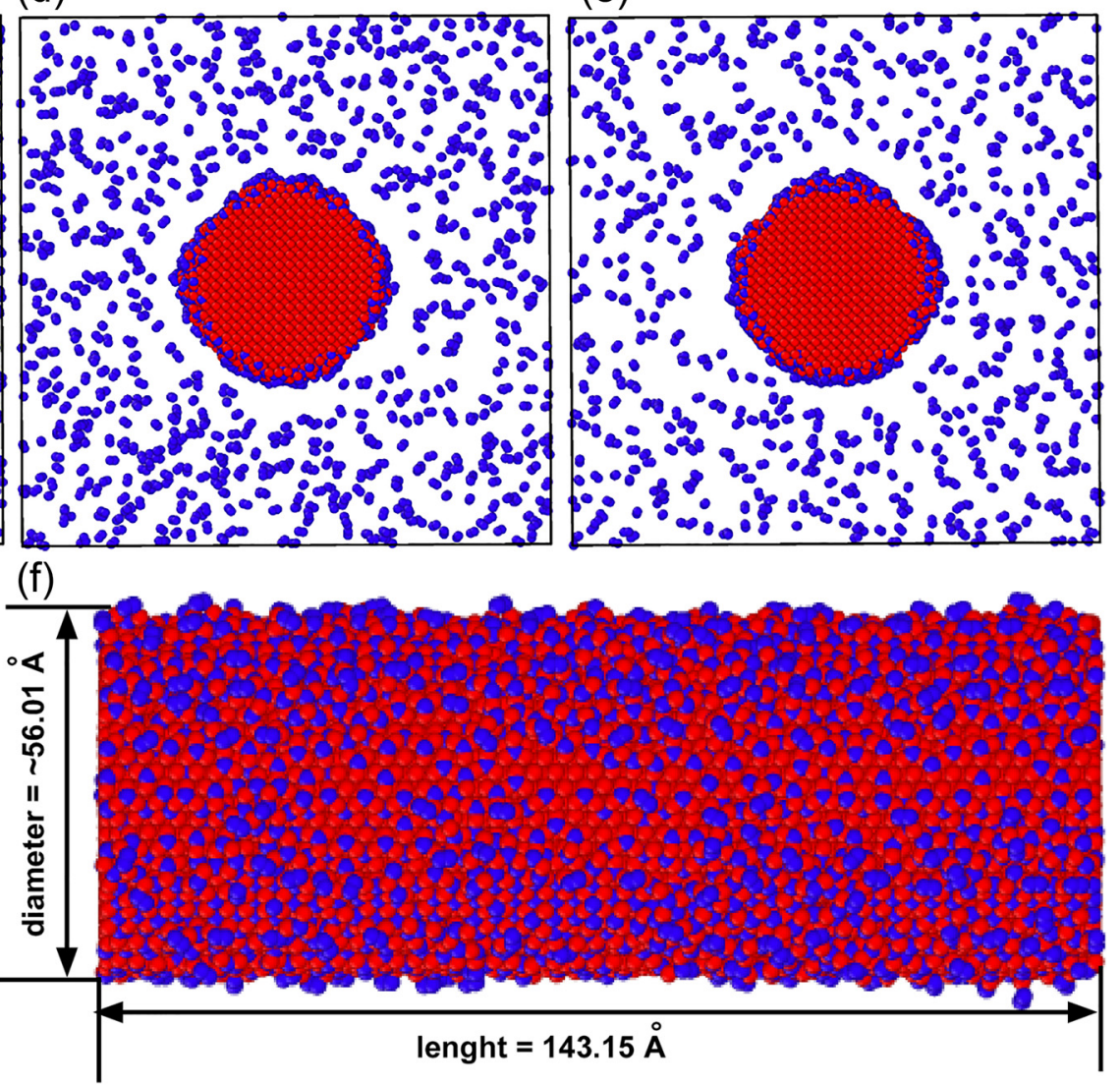

FIG. 1. Crystal structure of the initial cylindrical pure Fe NW with a $50.0 \AA$ diameter and $143.15 \AA$ length is shown from (a) sectional view and (b) side view along the axis. (c) The pure Fe NW is initially inserted in the $\mathrm{O}_{2}$ molecule environment. The oxide-coated cylindrical Fe NW with the $\mathrm{O}_{2}$ molecule environment is shown at (d) $3.3 \mathrm{~ns}$ and (e) $3.35 \mathrm{~ns}$. Upon completion of the oxidation process, the Fe-Fe $\mathrm{O}_{\mathrm{y}}$ core-shell structure is formed with $6.75 \AA$ thick native oxide shell layer on the pure Fe NW surface. The rough oxide shell layer has an average inner radius $\mathrm{r}=21.43 \AA$ and an average outer radius $\mathrm{r}=28.00 \AA$, as shown in (f) sectional view and (g) side view. (Red spheres represent Fe atoms and blue spheres denote $\mathrm{O}$ atoms.)

employed to integrate the equation of motion with a time step of 0.5 fs during all calculations. ${ }^{48}$ Atomic charges were determined at every MD time step in order to minimize the electrostatic potential energy, ${ }^{39}$ which were very time consuming. The total simulation time for the tensile test was $1.5 \mathrm{~ns}$.

In deformation simulations, the uniaxial constant strain rate of $0.01 \% \mathrm{ps}^{-1}\left(10^{8} \mathrm{~s}^{-1}\right)$ was repeatedly applied to all NWs by uniformly scaling their atomic coordinates along the [001] orientation in a stepwise manner until $16 \%$ of the initial box length was achieved. The applied strain rate was 8-12 orders of magnitude greater than the corresponding experimental value, confirming the need for further refinement of
MD simulations employed in studies of mechanical deformations of nano crystalline materials. The Virial theorem was used to obtain the stress-strain curve by averaging over all atomic stresses, ${ }^{48}$ before being corrected with the true volume of each individual Fe NW to obtain the corresponding engineering stress-strain curves.

\section{RESULTS AND DISCUSSION}

\section{A. Dynamic of oxide growth processes}

The simulation was run until 2870 oxygen atoms were consumed in the simulation of $3.35 \mathrm{~ns}$ duration. As a result, 


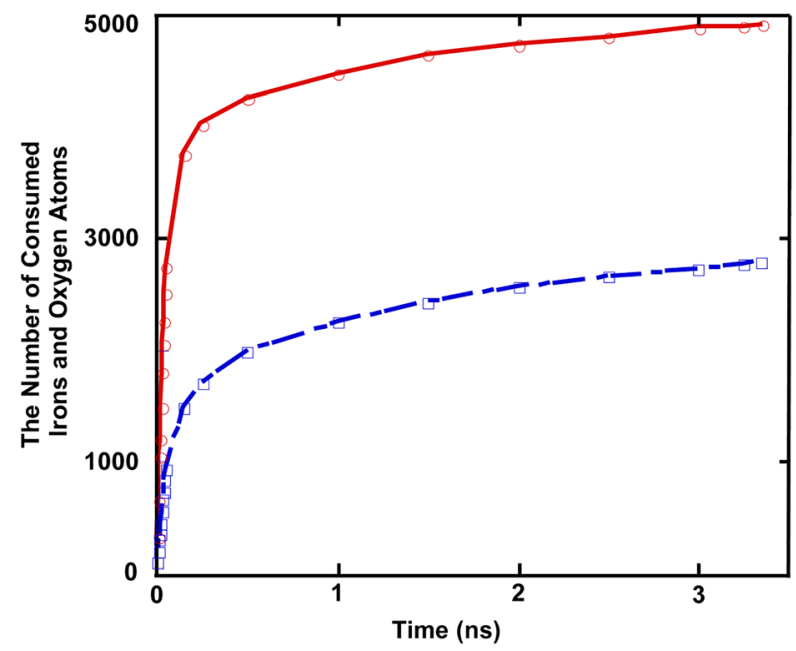

FIG. 2. Total number of oxygen and iron atoms consumed as a function of exposure time. Red and blue lines represent the consumption of $\mathrm{Fe}$ and $\mathrm{O}$ atoms, respectively.

the oxide-coated Fe NW consisted of an Fe core and an $\mathrm{Fe}_{\mathrm{x}} \mathrm{O}_{\mathrm{y}}$ surface shell layer. The dynamics of the oxide-shell growth are depicted in Figs. 2 and 3 (see supplementary material), which provide a physical picture of the reactivity of oxygen molecules on the Fe NW free surface and the oxidation growth rate as a function of simulation time, which can be strongly correlated with the number of consumed $\mathrm{O}$ and $\mathrm{Fe}$ atoms. Our simulation results reveal the existence of two successive oxidation stages: an initial rapid oxidation and a subsequent slow oxide growth. The latter stage has been typically characterized by a logarithmic growth law and was shown to be consistent with the Cabrera-Mott theory at low temperatures. ${ }^{1,2}$

Initially, oxygen molecules are strongly attracted by the Fe NW free surface, whereby O-O bonds are readily broken. Subsequently, they are decomposed into oxygen ions at the

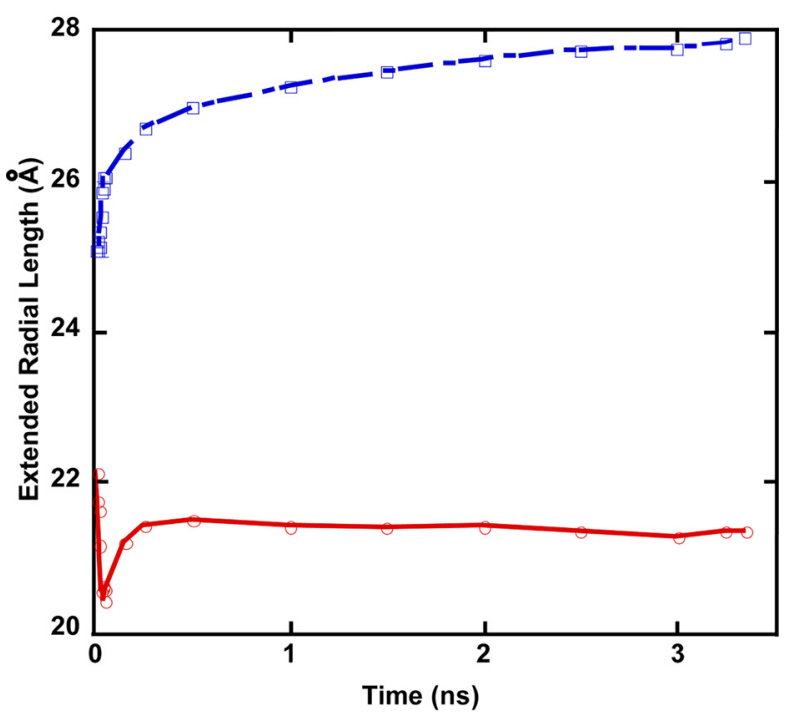

FIG. 3. The inner and outer oxide extent in the radial direction. In the process of Fe NW oxidation, the growth of the oxide layer is governed by the radial diffusion of both iron and oxygen atoms. Fe ions migrate outward, penetrating through the oxide scale, from the metal to the oxide layer surface. On the other hand, the oxygen ions migrate in the opposite direction. metallic Fe NW surface to form strong chemical bonds through complex chemical reactions, since $\mathrm{Fe}$ possesses great oxygen affinity. Oxygen ions instantaneously recombine with $\mathrm{Fe}$ atoms at the Fe NW free surface through electron transfer by maintaining overall charge neutrality in the simulated system based on the charge equilibration scheme. $^{45}$ Consequently, outward diffusion of $\mathrm{Fe}$ atoms through the developing oxide film and inward diffusion of $\mathrm{O}$ in the opposite direction are accompanied by transfer of electrons in the growing oxide layer. As a result of the formation of the bond between $\mathrm{Fe}$ and $\mathrm{O}$ atoms, the absolute averages of the valence charges increase from 0 to $\sim 0.59 \mathrm{e}$ for $\mathrm{O}$ atoms and $\sim 0.25 \mathrm{e}$ for $\mathrm{Fe}$ atoms in the developing oxide shell layer. The thickening of the oxide shell layer by inward diffusion of oxygen and outward diffusion of iron can be visualized and confirmed directly from Fig. 3, which provides detailed information on the kinetics of Fe NW oxidation, the total thickness of the oxide, the inner and outer extent, and the ratio of outward diffusion of iron to inward diffusion of oxygen as a function of time. In the initial stages of oxidation, substantial supply of oxygen atoms is required; however, the oxygen consumption rate gradually declines with increasing simulation time. ${ }^{2}$ At $3.35 \mathrm{~ns}, \sim 54.27 \%$ of the oxide growth $(\sim 3.57 \AA)$ can be attributed to the inward diffusion of $\mathrm{O}$ atoms, while $\sim 45.78 \%$ of the growth $(\sim 3.00 \AA)$ is due to the outward diffusion of $\mathrm{Fe}$ atoms. This total movement of $\mathrm{Fe}$ and $\mathrm{O}$ atoms leads to the formation of the oxide layer of $\sim 6.57 \AA$ thickness at $3.35 \mathrm{~ns}$ by expansion of the Fe core. Outward and inner diffusion of atoms control the overall oxidation growth rate. The outward movement of $\mathrm{Fe}$ is believed to correlate with the formation of wüstite and magnetite structures, whereas inward diffusion of $\mathrm{O}$ atoms forms the hematite local structure on the free surface. ${ }^{49}$ Consequently, after the oxidation process is completed, the average diameter of the pure Fe NW significantly increases, from $r=25.00 \AA$ to $r=28.00 \AA$, which has a great impact on the nanowire roughness and internal structure. The oxide shell layer on the Fe crystalline NW material surface produces large interfacial regions per unit volume and radial length, as expected. Moreover, oxidation of $\mathrm{Fe} \mathrm{NWs}$ gives rise to modifications in the size, reflected in $\sim 12 \%$ radial expansion and $\sim 25.4 \%$ volume expansion, resulting in $\sim 3.42 \%$ weight increment from the initial value characterizing the pure Fe NW. More than $\sim 34 \%$ of $\mathrm{Fe}$ and $\mathrm{O}$ atoms are associated with $6.57 \AA$ thick oxide shell layer at the interfacial boundary. In order to study the distribution of iron and oxygen atoms, we also calculated the average atomic mass density of the oxide shell layer as $\rho=4.63 \mathrm{~g} / \mathrm{cm}^{3}$ in the region between the inner radial distance (r) of $21.43 \AA$ and the outer radial distance of $28.00 \AA$ in the oxide shell layer. The average atomic mass density for the core Fe NW on the radial sections starting from the NW center consisting only of $17661 \mathrm{Fe}$ atoms was calculated as $\rho=8.04 \mathrm{~g} / \mathrm{cm}^{3}$, which is in good agreement with the iron bulk density of $7.874 \mathrm{~g} /$ $\mathrm{cm}^{3}$. Thus, the oxide surface formation, including some islands on the free surface and voids in the oxide shell layer, results in a lower mass density in the oxide shell layer.

The surface defects created by oxidation are normally accompanied by volume expansion. Consequently, the 
broken symmetry on the free surface exhibits drastic changes in comparison to the initial surface structure characteristics, including bonding types, surface roughness, shape, and diameter size. ${ }^{1,2}$ These structural changes in the Fe NWs due to progressive oxidation are particularly pronounced at the nanometer scale and have a great impact on the mechanical deformation mechanisms and related properties of core-shell NWs. In the present work, oxidation of Fe NWs in a molecular oxidizing environment leads to the formation of an oxide shell layer, in which the distribution of oxygen atoms is irregular along the entire NW length. More specifically, rapid outward diffusion of $\mathrm{Fe}$ and inward diffusion of $\mathrm{O}$ ions result in the formation of voids in the oxide shell layer. It is seen that the resultant rough oxide free surface with some oxide islands and small voids in the oxide shell layer changes the stoichiometry and local structure of the oxide shell layer. Therefore, the oxide shell layer generated on the free surface of metallic Fe NWs increases the Fe NW surface-to-volume ratio and causes surface defects during the oxidation process. These surface defects become more sensitive at all stresses below the ideal strength, ${ }^{50}$ which may help in overcoming the barrier for the onset of plastic deformation. According to Zhu et al., the size effect on the nucleation stress, for example, with a subtle variation in the wire geometry, directly changes the number of equivalent surface nucleation sites. ${ }^{51}$ Indeed, their mechanical deformation performance and related properties are expected to be different from those of their un-oxidized counterparts due to the defective structure of the oxide shell layer, which may act as the initiator for the early breaking of the oxide Fe NWs. To illustrate the effect of the oxide shell structure on the mechanical deformation mechanism, Sen et al. conducted an MD simulation for the $\mathrm{Al}$ NWs covered with crystalline and amorphous $\mathrm{Al}$ oxide shell layers. In this investigation, mechanical deformation behavior, as well as dynamics, was shown to be related to the structural type of the oxide shell layer. ${ }^{20}$

\section{B. Local structure characterization of the oxide shell layer}

In many scientific fields, there is a great demand for determining internal atomistic structural features of Fe oxide thin films. However, to date, this has not been successfully achieved, probably due to their complex structure as well as the presence of multiple coexisting phases. ${ }^{1,2}$ We have studied the order of the short-range structural correlations in the final oxide shell layer using the partial radial distribution function (RDF), coordination numbers, and bond-angle distributions. The oxide shell layer formed on the pure Fe NW has unique structural properties, including density, bond structure, and stoichiometry, which are strongly dependent on the depth. ${ }^{1,2}$ The developed oxide shell layer comprises $6389 \mathrm{Fe}$ and $2783 \mathrm{O}$ atoms in the short-range order by forming the stoichiometry of the oxide $\mathrm{Fe}_{\mathrm{x}} \mathrm{O}_{\mathrm{y}}$ layer with $\mathrm{y} / \mathrm{x}$ $\cong 0.43(\mathrm{x} / \mathrm{y} \cong 2.29)$. Subbaraman et al. showed the depth dependence of the structural characteristics and the stoichiometry of $\mathrm{O} / \mathrm{Fe}$ atom numbers, revealing that they are strongly correlated with the oxygen pressure and crystal orientation. ${ }^{1}$ For example, in the (110) crystal orientated oxide metallic Fe at high pressure, $\mathrm{O} / \mathrm{Fe}$ stoichiometry in the oxide region varies from $\sim 1.4$ at the oxide-gas interface to $\sim 0.6$ in the oxide interior. Based on the RDF, the first peaks in the Fe-O pair split into two peaks, centered at $\sim 1.6$ and $\sim 2.05 \AA$, respectively. The results are clearly shown in Fig. 4. The first peak is also slightly sharper and has the same amplitude compared with the second peak. The $\mathrm{Fe}-\mathrm{Fe}$ bond length is located at $2.6 \AA$ and the $\mathrm{O}-\mathrm{O}$ partial RDF has a sharp peak located with maximum amplitude located at $1.35 \AA$. The peak presumably corresponds to the distances between the oxygen atoms from the un-dissolved oxygen molecules at the oxide shell layer, even though most of the $\mathrm{O}$ atoms are segregated out from the oxygen molecules. The second intense peak for the $\mathrm{O}-\mathrm{O}$ pair occurs at $3.2 \AA$. Jeon et al. studied the pair distributions of $\mathrm{Fe}-\mathrm{O}$ as a reference for three different $\mathrm{Fe}$ oxide states-wüstite, magnetite, and hematite - at low and high temperatures, aiming to compare their developed oxidized layers. ${ }^{2}$ Among these oxides, hematite is the most common form for developing iron oxide layers. For low temperature conditions, the $\mathrm{Fe}-\mathrm{O}$ bond length is dominant, and its peak is located at around $1.65 \AA$ for wüstite. On the other hand, for magnetite, the main peak is estimated at $2.0 \AA$, but a slight transition is observed at 1.6 $\AA$. Hematite shows a distinct transition at $2.15 \AA$, while the main peak is located at $1.7 \AA$. The average $\mathrm{Fe}-\mathrm{O}$ bond length in the oxide structure results in a dominant peak at $1.5-1.6 \AA$, which is a slightly shorter distance than that reported for the reference oxides studied. Subbaraman et al. have also shown similar structural features for wüstite, magnetite, and hematite oxides. ${ }^{1}$ Their radial distribution function of the $\mathrm{Fe}-\mathrm{O}$ for the reference $\mathrm{Fe}$ oxide structures shows that there is one peak $\sim 1.65 \AA$ for wüstite and $\sim 1.8 \AA$ for the magnetite structure. Hematite shows a distinct peak at $\sim 1.7 \AA$ and a prominent shoulder at $\sim 2.1 \AA$. Possibly, our result for the bond length of $\mathrm{Fe}-\mathrm{O}$ peaks at $\sim 1.6 \AA$ and $\sim 2.05 \AA$ is an indication of formation of both wüstite and hematite structures. In addition, the average $\mathrm{Fe}-\mathrm{O}$ bond length exhibits a peak around $1.8 \AA$ for all the surface oxides

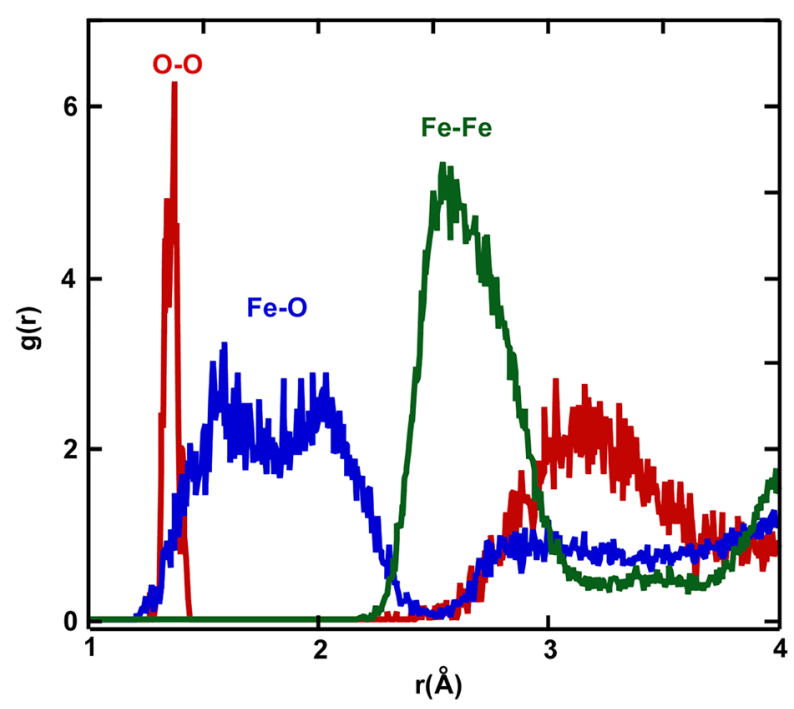

FIG. 4. Radial pair distribution function $\mathrm{g}(\mathrm{r})$ for $\mathrm{Fe}-\mathrm{O}$ (blue), O-O (red), and $\mathrm{Fe}-\mathrm{Fe}$, in the shell of the oxide region, is shown. 
studied. Those results show distinct differences among the tested iron oxide states. In our calculation, the bond length of $\mathrm{Fe}-\mathrm{O}(1.6 \AA)$ is in close agreement with those of the Jeon simulation results for the developed oxide, wüstite, and magnetite structures. On the other hand, the second $\mathrm{Fe}-\mathrm{O}$ structure peak $(2.05 \AA)$ is located at a slightly shorter distance of $2.15 \AA$ than that for the distinct transition of the hematite structure, reflecting the coexistence of various bonds. Still, the $\mathrm{Fe}-\mathrm{O}$ bond length in amorphous hematite is characterized by $1.91 \AA^{52}$ In the experimental structural studies of the iron oxide shells using nano-Raman spectroscopy, the structural data revealed that magnetite is the major constituent. ${ }^{14}$ The longer bond length of the $\mathrm{Fe}-\mathrm{O}$ pair $(\sim 2.05 \AA)$ is almost the same as $2.008 \AA$ for the surface parts of sodium dodecyl benzene sulphonate (DBS) coated hematite nanoparticles and bulk materials. ${ }^{53}$ However, there are some difficulties when studying the iron oxide film. For example, in the XPS study, the iron-oxide film is very vulnerable to external stimulation and easily changes its phase.

The pair distribution function of the $\mathrm{Fe}-\mathrm{Fe}$ atoms in the core region before the applied tensile test as well as before the onset of the plastic deformation is shown in Fig. 5. Prior to the applied tensile test, radial distribution functions of the system (Fig. 5) show that there are two peaks for the pair $\mathrm{Fe}-\mathrm{Fe}$ at $2.45 \AA$ and $2.85 \AA$ in the core region. On the other hand, the first and second peaks in RDF for the bond length of Fe-Fe occur at $2.5 \AA$ and $2.75 \AA$ before the onset of the plastic deformation. Moreover, the second peak splits into two peaks. With respect to Fe-Fe pair interactions, Jeon et al. observed two similar peaks at 2.45 and $2.80 \AA$ for the $\mathrm{Fe}(100)$ oriented surface at $900 \mathrm{~K}$.

Integration $\mathrm{PDF}$ of the $\mathrm{Fe}-\mathrm{O}$ pairs up to $2.4 \AA$ yields a total mean coordination number of 1.55 . The average distribution of oxygen atoms around the iron atoms is characterized by the following frequencies: $\mathrm{Z}_{\mathrm{FeO}}=1$ (39\%), $\mathrm{Z}_{\mathrm{FeO} 2}=2(29 \%), \mathrm{Z}_{\mathrm{FeO} 3}=3(23 \%)$, and $\mathrm{Z}_{\mathrm{FeO} 4}=4(7 \%)$, while the remaining $1 \%$ represents the contribution to the total mean coordination number in the oxide shell region. It

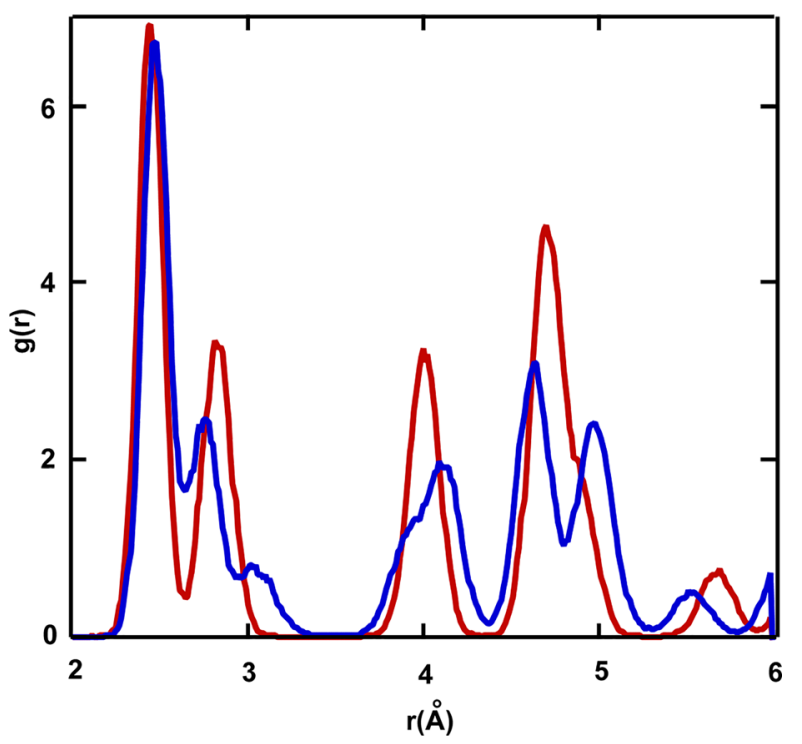

FIG. 5. Fe-Fe radial pair distribution functions before the applied tensile test (red line) and before the onset of the plastic deformation (blue line) in the core region. is noted that the local structure of the oxide shell layer is predominantly a mixture of 1-fold $(\mathrm{FeO}), 2$-fold $\left(\mathrm{FeO}_{2}\right)$, and 3 -fold $\left(\mathrm{FeO}_{3}\right)$ coordinated structures, whereas 1 -fold $\mathrm{FeO}$ is the dominant structure in the oxide shell layer. However, a 5 -fold coordinated iron atom with $\mathrm{O}$ atoms is rarely found in the oxide shell. On the total main coordination number, $\mathrm{Fe}$ atoms were coordinated to $1.55 \mathrm{O}$ atoms and $\mathrm{O}$ atoms to 3.6 $\mathrm{Fe}$ atoms, consistent with the $\mathrm{O} / \mathrm{Fe}$ ration of 0.43 . In addition, immediately prior to the onset of plastic deformation, we calculated the mean coordination number of $\mathrm{Fe}-\mathrm{O}$, which was slightly reduced from 1.55 to 1.53 . Nonetheless, very little change occurred in the frequencies, i.e., $\mathrm{Z}_{\mathrm{FeO}}=1$ $(38 \%), \mathrm{Z}_{\mathrm{FeO} 2}=2(30 \%), \mathrm{Z}_{\mathrm{FeO} 3}=3(25 \%)$, and $\mathrm{Z}_{\mathrm{FeO} 4}=4$ $(5 \%)$, with the rest contributing to the remaining $2 \%$.

The Fe-O-Fe bond angle distribution has a broad peak at around $100^{\circ}$. On the other hand, the O-Fe-O bond angle distribution has a peak at $87^{\circ}$ and $120^{\circ}$, whereas the oxide shell layer structure is strongly dependent on the depth of the oxidation shell layer as well as temperature. ${ }^{1,2}$ This means that two peaks of $\mathrm{O}-\mathrm{Fe}-\mathrm{O}$ angle distribution indicated a distorted octahedral network structure in the system. ${ }^{52}$ These values are close to the O-Fe-O angles of $80^{\circ}$ and $94^{\circ}$ and the Fe-OFe angles of $97^{\circ}$ and $120^{\circ}$ for amorphous hematite with a density of $5.19 \mathrm{~g} / \mathrm{cm}^{3}$ at $350 \mathrm{~K}$, as reported by Khanh et al. ${ }^{52}$ It is well-known that for an ideal octahedron, the $\mathrm{O}-\mathrm{Fe}-\mathrm{O}$ angle is equal to $94^{\circ}$, and for an ideal tetrahedron, it is equal to $109.47^{\circ}$. This means that two peaks of $\mathrm{O}-\mathrm{Fe}-\mathrm{O}$ angle distribution indicated a distorted octahedral network structure in the system. ${ }^{52}$ In a recent experimental study, the authors reported that oxidation with oxygen molecules of metallic $\mathrm{Fe}$ leads to the formation of oxide layers in a sequential order, resulting in a mixture of two or three types of $\mathrm{Fe}$ oxides, namely, hematite, magnetite, and wüstite structures at temperatures above $570{ }^{\circ} \mathrm{C}^{50}$ The hematite and wüstite layers occupy the outermost region and the innermost region, respectively, whereas the magnetite layer was observed in between these layers. ${ }^{19}$

\section{Effect of oxide shell layers on stress-strain behavior with plastic properties}

Engineering of a stress-strain behavior with and without the existence of a native oxide layers in the [001] direction revealed a distinct feature, which is characterized by three stages, namely, linear elasticity, nonlinear elasticity, and plasticity deformation stages, as shown in Fig. 6. In a recent experimental study, Landau et al. reported qualitatively similar three stages in the stress-strain curves for Fe NWs, in which the stress-strain curve for the as-fabricated and implanted nano Fe pillars is characterized by elastic loading as Stage I, substantial strain hardening as Stage II, and steady state discrete plasticity where the average flow stress remains unchanged as Stage III. ${ }^{25}$ Consequently, all simulated $\mathrm{Fe}$ NWs exhibit similar stress-strain behavior to those obtained experimentally for bcc Fe nano pillars.

The stress-strain curves of the pure Fe NW in Stage I show a linear increase up to a yield strain of $6.0 \%$, whereas this deformation is limited to $3.5 \%-5.0 \%$ for oxide-coated Fe NWs. In Stage II, the strain values are between 6.0\% and 


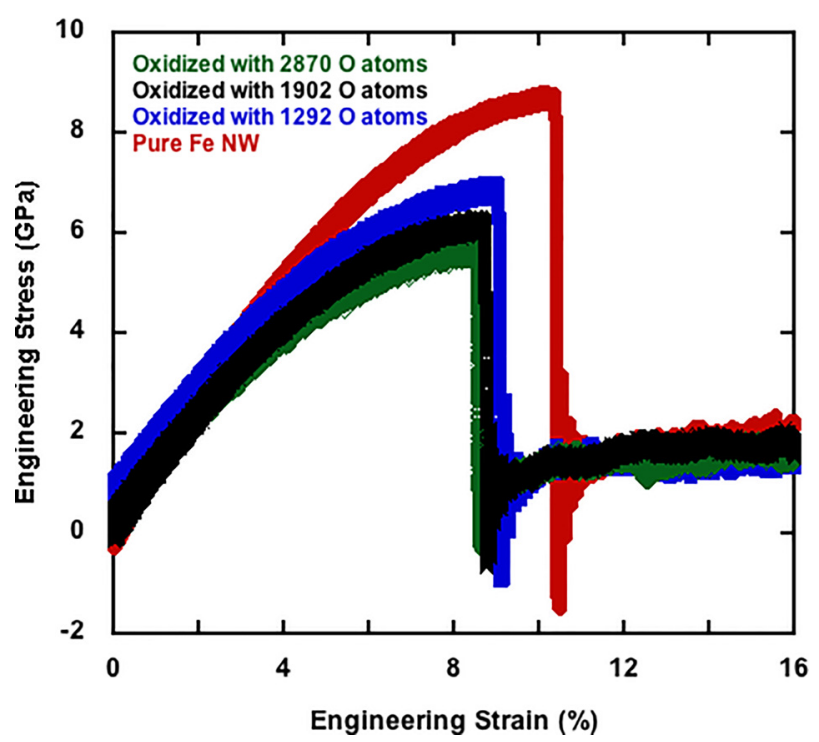

FIG. 6. Engineering stress-strain curves for pure and oxide bcc Fe NWs at $300 \mathrm{~K}$.

$10.4 \%$, and the stress-strain curves exhibit obvious nonlinear character, reaching the maximum stress value just prior to the nucleation of a partial dislocation, as shown in Fig. 6. Further increases in the strain beyond the maximum critical stress level of $8.7 \mathrm{GPa}$ at $10.4 \%$ strain lead to an abrupt decline in the stress profile down to $0.0 \mathrm{GPa}$, which indicates the onset of plastic deformation. Immediately after that, the flow stress rapidly increases toward the steady-state level of $\sim 1.82 \mathrm{GPa}$ through a twinning deformation, referred to as Stage III, described in more detail below. The linear yield and maximum critical stresses for the pure Fe NW are estimated at $6.82 \mathrm{GPa}$ at $6.0 \%$ linear strain and $8.7 \mathrm{GPa}$ at $10.4 \%$ critical strain, respectively. The same tendency is also observed for the oxide-coated Fe NWs; however, the native oxide layer around the Fe NWs is associated with significant reductions in the NW strength, and thus its potential for lowering the onset of plastic deformation with increasing oxide layer thickness. Stage I of oxide-coated NWs is linearly correlated with strain values up to $\sim 3.5 \%, \sim 4.0 \%$, and $\sim 5.0 \%$, which correspond to the yielding linear stress at approximately $3.90 \mathrm{GPa}, 4.20 \mathrm{GPa}$, and $5.42 \mathrm{GPa}$ for $\sim 6.57 \AA$, $\sim 5.33 \AA$, and $\sim 4.81 \AA$ oxide shell layer thicknesses, respectively. Stage II, stress values gradually increase from $3.90 \mathrm{GPa}$ at $\sim 3.5 \%$ of linear strain to $5.42 \mathrm{GPa}$ at $\sim 5.0 \%$. A maximum critical tensile strength also increases from $5.7 \mathrm{GPa}$ at $\sim 8.55 \%$ of critical strain to $6.9 \mathrm{GPa}$ at $\sim 9.05 \%$ for increasing oxide shell layer thicknesses. This indicates that the oxide shell layer thickness affects the mechanical properties of NWs. Once the maximum critical stresses are reached, defect formation begins. A sharp decline in stress occurs due to further loading, which causes the onset of the Stage III, with the remaining constant average flow stress at approximately $\sim 1.41-1.59 \mathrm{GPa}$ for the oxide-coated NWs. Importantly, the similarity of flow stress responses for all NWs indicates that the plastic deformation mechanism in all cases must be similar. Our results are consistent with those obtained in Landau's experimental study for the pure iron nano-pillars. $^{25}$ Landau et al. distinguish the stress-strain curve into three stages, whereby Stage I denotes elastic loading up to a strain of $\sim 2 \%$, while Stage II pertains to the maximum tensile strength at a strain of $3 \%-5 \%$, and estimate the maximum critical yield stress at $1.45 \mathrm{GPa}$, and Stage III represents a steady state region where the average flow stress remain nearly constant for the as-fabricated samples. These results are well aligned with those obtained for the oxidecoated Fe NW with a $6.57 \AA$ oxide layer thickness. There are two sets of $\langle 101\rangle$-oriented cylindrical single crystalline iron nano-pillar samples with diameter $100 \mathrm{~nm}$ : one is unmodified, which refers "as-fabricated" and the other is modified by implanting with helium throughout gauge sections. The difference may be due to the orientation and diameter of the as-fabricated cylindrical Fe nano-pillars and the initial defect structure. Zhang performed a tensile deformation test for the $\mathrm{Fe}$ nano-pillar oriented along [001] directions at a $\mathrm{T}=300 \mathrm{~K}$, reporting the corresponding critical stress and strain of $3.39 \mathrm{GPa}$ and $11.8 \%$, respectively. ${ }^{54}$ The theoretical calculations of tensile strengths for bcc Fe predict the values of $12.4,32.7$, and $27.5 \mathrm{GPa}$ in the [100], [110] and [111] directions, respectively, depending on the NW orientation. ${ }^{55}$ Similarly, the Young's modulus for bulk Fe was observed to be $131 \mathrm{GPa}, 219 \mathrm{GPa}$, and $283 \mathrm{GPa}$ in the [100], [110], and [111] directions, respectively. ${ }^{56}$

Table I presents the elastic moduli as well as other elastic properties of Fe NWs as a function of oxide layer thickness. The Young's modulus is $114.5 \mathrm{GPa}$ with a Poisson's ratio of 0.33 for the pure $\mathrm{NW}$, whereas the modulus due to the coupling with the oxide layer decreases to $94.1 \mathrm{GPa}$ with a Poisson's ratio of 0.42 for the oxide-coated Fe NW with the shell layer of $6.57 \AA$ thickness. These results indicate that the oxide layers cause significant degradation in the elastic properties of the pure Fe NW, thus reducing the mechanical deformation resistance. It should be noted that the thickest oxide layer reduces the Young's modulus by about $\sim 17.8 \%$. Adams et al. discussed the effect of temperature on the elastic constants for monocrystalline $\mathrm{Fe}$ over the temperature

TABLE I. Mechanical properties of the pure and oxide-coated Fe NWs with different shell layer thicknesses at constant strain rate. The values of the thickness of oxide shell layer (thickness), linear yield strain $\left(\varepsilon_{\mathrm{z}}{ }^{\text {lin }}\right)$, linear yield stress $\left(\sigma_{\mathrm{z}}^{\text {lin }}\right)$, maximum critical yield stain $\left(\varepsilon_{\mathrm{z}}{ }^{\text {lin }}\right)$, maximum critical stress $\left(\sigma_{\mathrm{z}}^{\text {lin }}\right)$, and Young's modules (E) are shown. Three different (1292, 1902, and 2870) numbers of oxygen atoms are involved in the formation of the oxide shell layers.

\begin{tabular}{|c|c|c|c|c|c|c|}
\hline System & Thickness (§) & $\varepsilon_{\mathrm{z}}^{\operatorname{lin}}(\%)$ & $\sigma_{\mathrm{z}}^{\operatorname{lin}}(\mathrm{GPa})$ & $\varepsilon_{\mathrm{z}}^{\max }(\%)$ & $\sigma_{\mathrm{z}}^{\max }(\mathrm{GPa})$ & $\mathrm{E}(\mathrm{GPa})$ \\
\hline Pure & & 6.0 & 6.82 & 10.40 & 8.7 & 114.8 \\
\hline 1292 oxygen atoms involved in oxidation & 4.81 & 5.0 & 5.42 & 9.05 & 6.9 & 97.6 \\
\hline 1902 oxygen atoms involved in oxidation & 5.33 & 4.0 & 4.20 & 8.75 & 6.2 & 100.9 \\
\hline 2870 oxygen atoms involved in oxidation & 6.57 & 3.5 & 3.90 & 8.55 & 5.7 & 94.1 \\
\hline
\end{tabular}


range of 3 to $500 \mathrm{~K}$ by using resonant ultrasound spectroscopy. ${ }^{57}$ At low temperatures, the Young's modulus $\mathrm{E}_{100}$ and Poisson ratio $\nu_{100}$ were estimated to be $131.73 \mathrm{GPa}$ and -0.3679 , respectively, which are in line with our results.

The local shear strain and stress distributions on the surfaces were proved to be a significant factor in controlling the dislocation nucleation. ${ }^{58}$ The stress concentration in some regions on the free surface is much larger than the average stress inside the Fe NW. Such high stress regions will behave eventually themselves as both sources and sinks for the future nucleation of dislocations. Before the onset of the plastic deformation, the local stress concentrations on the free surface greatly increase with increasing strain until they reach a critical value. When the loading exceeds the bond strength, then $1 / 6\{112\}\langle 111\rangle$ Shockley partial dislocation simultaneously nucleates from the free surfaces of wires. ${ }^{27}$ At this point, the local stress is relieved by emission of dislocations, which acts to reduce the overall free energy of the system; and thus the nucleation barrier for dislocation initiation becomes lower. Furthermore, beyond the maximum stress, the stress decreases abruptly despite the increasing strain, which is considered as the onset of plasticity. These processes are driven predominantly by a combination of the applied stress and local stress relaxation within the free surface. The dislocations transform into a twin nucleus, through a series of dislocation emission steps, which leads to accommodate strains that develop during plastic deformation. Thus, the nucleation of twins with twin boundary and an alteration in twinning propensity mechanisms have been predominantly connected with an alteration in stress-strain response of the all Fe NWs.

Our results demonstrate that the unique features of oxide shell layers have a direct effect on the elastic mechanical properties of the cylindrical metallic Fe NWs. Reduction in the elastic strength of the Fe NW, the Young's modulus, linear yield strain, linear yield strength, maximum critical strain, and strength of the Fe NWs are significantly sensitive to the oxide layer thickness. The oxide defect shell layer may act as a productive source of dislocations, thus reducing the onset of plastic deformations. Therefore, plastic deformation in the oxide-coated Fe NW is easily initiated as compared to the pure Fe NW. Hence, comparatively low flow stress is sufficient for all $\mathrm{Fe}$ NWs plastic deformations to continue in a ductile manner at the remaining average constant stress levels beyond a maximum stress through twinning. There are relatively small distinguishable differences in the attained flow stresses between the pure and oxidecoated Fe NWs. Our results are consistent with those yielded by the previous MD simulation studies of plasticity for $\mathrm{Fe}$ nano pillars. For example, while large stress is initially required to create the twins, the strain subsequently continues at an almost constant value as the twin boundaries expand. ${ }^{28}$ The maximum yield strength and flow stress value of the pure Fe NWs are also consistent with those reported by Li et al., who showed that the yield stress of pure Fe NW is around $\sim 10.0 \mathrm{GPa}$. Consequently, the stress declines dramatically to a relatively low value and further loading causes an extensive plateau stage, whereby stress remains at approximately $\sim 1.9 \mathrm{GPa}^{31}$ Landau also reported that flow stress beyond 5\% strain fluctuates at a nearly constant stress of $1.7 \mathrm{GPa}$, which indicates that dislocation generation and annihilation occur at a comparable rate. ${ }^{25}$

\section{The role of oxidation in the tensile deformation behavior of Fe NWs}

The complete process of plastic mechanical deformation with twinning for all Fe NWs roughly occurs in three stages, denoted as nucleation, propagation, and thickening. In the present study, the $\mathrm{Fe}_{\mathrm{x}} \mathrm{O}_{\mathrm{y}}$ shell layer remained fully bonded onto the $\mathrm{Fe}$ core and did not exhibit any cracks during the tensile loading. The onset of plasticity for the [001]-oriented pure and oxide-coated Fe NWs, promoted by specifically nucleation through a/6 $\langle 111\rangle$ Shockley partial dislocation on the $\{112\}$ glide planes from the energetically favorable edge on the free surface of the cylindrical $\mathrm{NW},{ }^{31}$ is clearly seen from the stress-strain curve shown in Figs. 7 and 8 and is associated with a sharp decline in stress from the maximum critical yield stress to $0.0 \mathrm{GPa}$. However, for the oxidecoated Fe NWs, yielding occurs at relatively lower strain and stress values compared to those of the pure NW, i.e., the defects due to the oxide shell layer thickness increase and the mechanical resistance to plastic deformation decreases. When the applied strain is increased further, the $a / 6\langle 111\rangle$ Shockley partial dislocation on the $\{112\}$ glide plane starts to propagate laterally toward the other NW surface along the cross-sectional length, and the new partial dislocation quickly emerges from the next parallel $\{112\}$-glide plane. ${ }^{31}$ Surprisingly, the crystallographic lattice reorientation immediately begins with the formation of these two partial dislocations. Consequently, a complete and unique abrupt structural reorientation from [001] to [110] driven defect is observed between these two partial dislocations. Moreover, nucleation of two close and identical partial dislocations is activated on the $\{112\}$-glide plane in the $\langle 111\rangle$ direction without the influence of native oxide layer effects, as shown in Figs. 7 and 8 (supplementary material, Fig. 1). As a result of consecutive continuous motion of partial dislocations from one surface toward the opposite side on the adjacent $\{112\}$ glide planes, the partial dislocations finally coincide at the opposite surface of the NWs, where they are annihilated, leaving behind a pair of stacking faults with continued tensile loading. ${ }^{31}$ The common neighbor analysis (CNA) technique with the visualization package tool OVITO was employed to accurately identify and characterize the NW defect structure. ${ }^{59}$ Defects associated with the local crystalline disordered atoms, distributed at the surface, dislocation core, and twin boundaries, are clearly shown in Figs. 7 and 8. In these images, all atoms were colored according to their specific local structural types and were visualized by CNA. Blue and red colors represent perfect bcc atoms and unknown atoms, respectively.

Three preferred primary dislocation slip systems in bcc iron metal are of type $\{110\}\langle 111\rangle,\{112\}\langle 111\rangle$, and $\{123\}\langle 111\rangle$; however, their activation strongly depends on the crystallographic loading axis, crystal purity, and experimental factors. ${ }^{60}$ At the atomistic level, defect nucleation in bulk crystal materials has to overcome significant resistance 

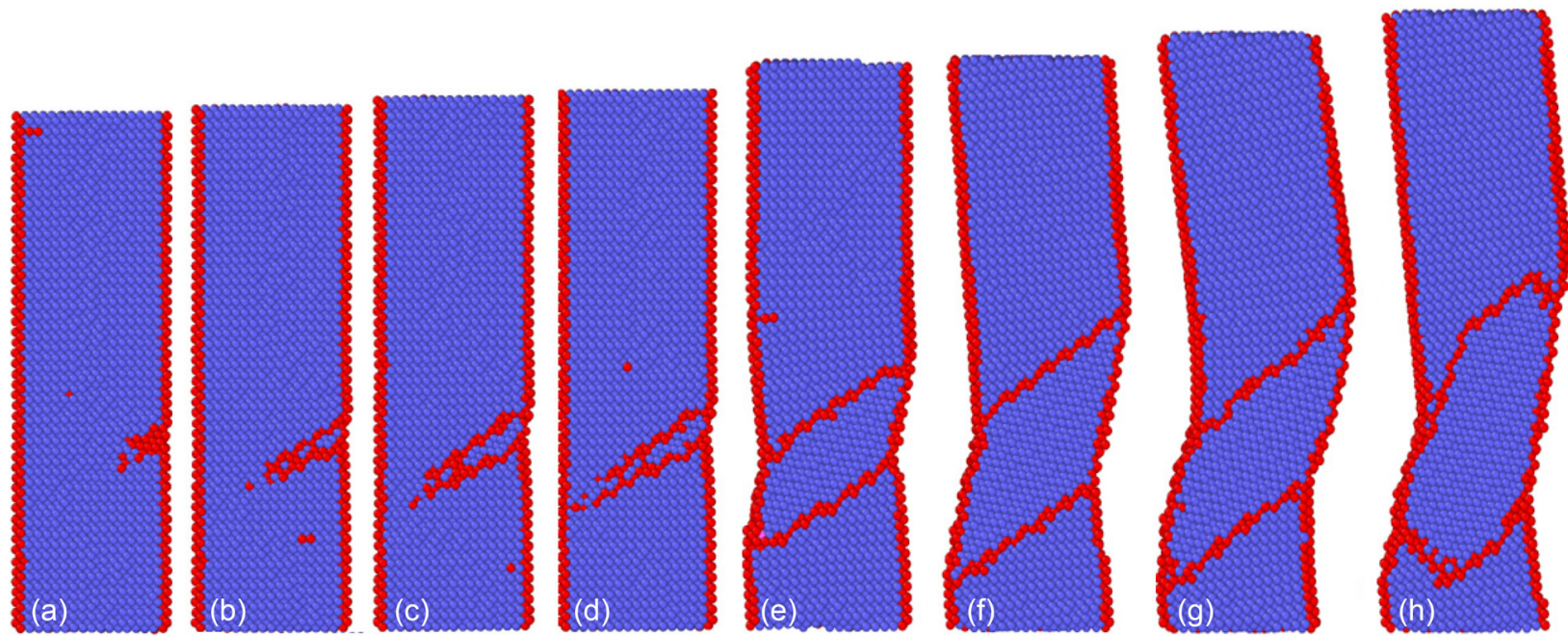

FIG. 7. Perspective view of the half-slices at different stages of deformation shows initiation nucleation, evolution, and propagation of the twin for the cylindrical pure Fe NW at (a) $10.3777 \%$, (b) $10.3805 \%$, (c) $10.3838 \%$, (d) $10.3843 \%$, (e) $10.4014 \%$, (f) $10.4566 \%$, (g) $10.8437 \%$, and (h) $16.0 \%$ strain. Atoms are colored according to the local crystalline structure, determined by common neighbor analysis methods (CNA). The blue spheres denote atoms with the bcc structure, whereas the red ones correspond to the unknown structure. Defects associated with the local crystalline disordered atoms are colored red, whereby more disordered atoms are distributed at the surface and at twin boundaries. The only observed deformation mechanism is twinning, which is generated at lower strain and then propagates along the nanowire length under further tensile loading.

to propagate across the gliding plane - a mechanism that is quantitatively predicted by the Schmid factor. The value of that associated with [001] tensile loading for bcc Fe is approximately 0.47 and 0.41 on the $\{112\}$ as the primary and $\{110\}$ as the secondary defect activation planes in $\langle 111\rangle$ directions, respectively. ${ }^{60}$ Our results demonstrate that the dislocation activity occurs in the most preferred $\{112\}\langle 111\rangle$ primary slip system in both pure and oxide-coated Fe NWs, according to the dynamic process, as reported in a similar molecular dynamics simulation by Li et al. Our results are also consistent with the proposed Schmid law. ${ }^{31}$ However, bcc materials do not obey Schmid's law in most cases. Thus, in the experimental studies of bcc Fe pillars, dislocations caused by compressive force are activated more dominantly along the slip $\{110\}$ planes instead of the expected $\{112\}$ planes. ${ }^{60}$

Although partial dislocations propagate over the same $\{112\}\langle 111\rangle$ slip system, the time required for the migration process-from the onset of the initial partial dislocation to finally reaching full straight twin boundary on the $\{112\}$ glide plane-is measured at $\sim 2.0 \mathrm{ps}$ and $\sim 5.3 \mathrm{ps}$ for the pure and oxide-coated Fe NWs, respectively. However, comparing the cross-slip partial dislocation propagation speeds for NWs with and without the oxide shell layer, the oxide shell layer impedes the escape of initial partial dislocation, which increases as the oxide shell layer thickness decreases. These dislocation activities may contribute to the overall plastic deformation and the mechanical strength of the $\mathrm{Fe}$
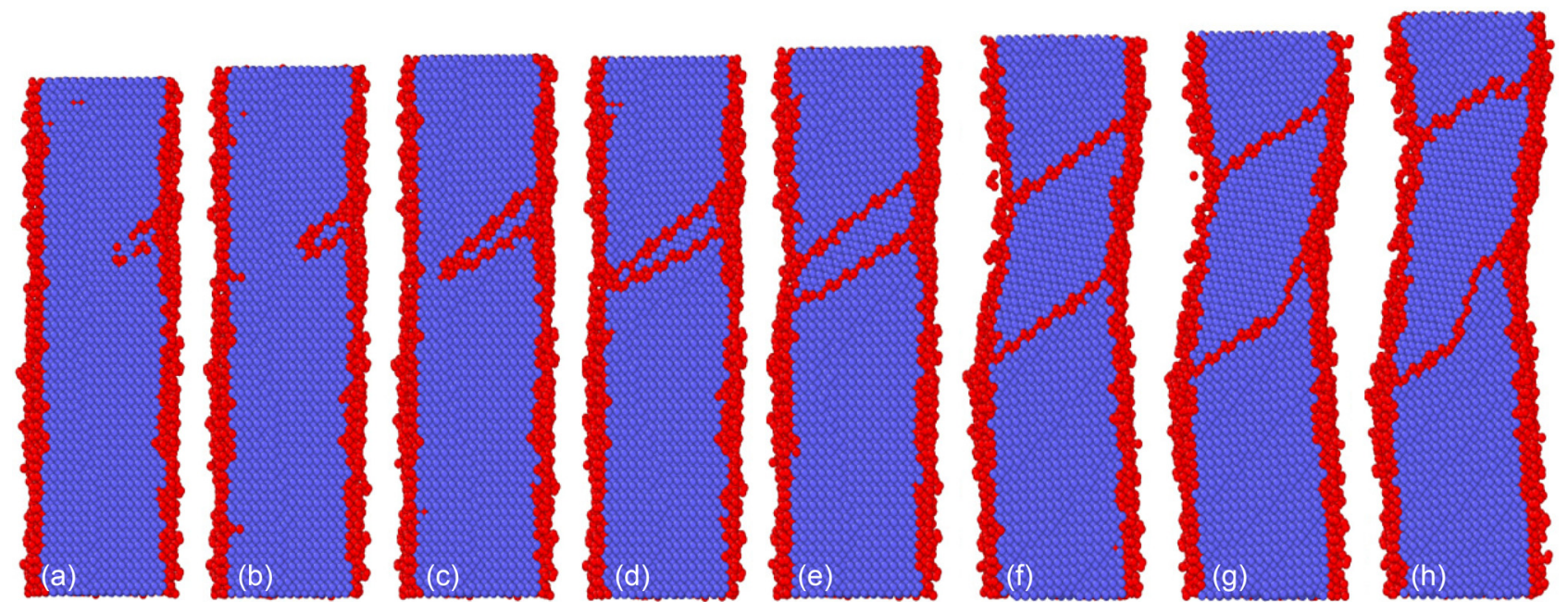

FIG. 8. Twinning is also the only plastic deformation mechanism for the oxide-coated NWs during tensile deformation. Perspective view of the half-slices shows defect evolution of the twinning boundaries inside the oxide cylindrical Fe NW with $6.57 \AA$ thickness layer at (a) $8.5159 \%$, (b) $8.5175 \%$, (c) $8.5267 \%$, (d) $8.5289 \%$, (e) $8.5359 \%$, (f) $10.5118 \%$, (g) $12.6305 \%$, and (h) $16.0 \%$ strain under tensile loading along the nanowire axis. The partial dislocation nucleates from the surface of the oxide Fe NW and moves along the $\{112\}$-slip plane in the $\langle 111\rangle$ direction, eventually creating twinning. At low strain, twin boundaries are parallel. However, as the strain increases further, they become curved because of the activation of numerous slip planes. 
NWs through a decrease in the partial dislocation slip propagation rate, thereby softening the oxide Fe NWs. The collective nature of velocity differences may be strongly related to the variation in the critical resolved shear stress (CRSS), because the maximum resolved shear stress values are significantly different from each other. The Shockley partial dislocations propagated by gliding on the $\{111\}\langle 112\rangle$-slip system are caused by the CRSS, which is given by $\tau_{\mathrm{R}}=\sigma_{\mathrm{z}}{ }^{*}$ (Schmid factor), where $\sigma_{\mathrm{z}}$ is the magnitude of the maximum critical yield stress. ${ }^{54}$ The calculated CRSS, required for the initiation of plastic deformation, is $\sim 4.1 \mathrm{GPa}$ and $\sim 2.7 \mathrm{GPa}$ for the pure and oxide-coated Fe NWs, respectively. The relatively higher CRSS value for the pure Fe NW relative to the oxide-coated $\mathrm{Fe} \mathrm{NWs}$ provides an energetic explanation for the dislocation propagation. Due to the large differences between the CRSS values, it can be assumed that the partial Shockley dislocations move more rapidly across the $\{112\}$ glide plane in the $\langle 111\rangle$ direction in the pure NW than in the oxidized NW. Therefore, the oxide shell layer has the ability to control initial motion of dislocation nucleation inside the cylindrical Fe NWs, which is a unique feature of the Fe NW plastic deformation mechanism. In terms of the initial migration speed of a partial dislocation, Sen and colleagues observed a similar phenomenon for the oxide $\mathrm{Al} \mathrm{NWs,} \mathrm{which}$ is consistent with our simulation results. ${ }^{21}$ Sen $e t$ al. successfully applied the image forces method associated with longrange elastic interactions and surface oxide defects to explain velocity differences of partial dislocations in the Al NWs. Thus, they demonstrated that the surface amorphous oxide shell layer of Al NWs would hinder the dislocation escape velocity.

After the formation of one atomic mobile twin boundary layer, both pure and oxide-coated Fe NWs undergo plastic deformation by twinning. During this process, the twin boundary planes diverge laterally towards the nanowire ends layer by layer, through sequential activation of partial dislocations. Thus, collective movement of these dislocations as a result of increasing the applied strain leaves behind surface and lattice reorientations between the existing twin boundaries during the plastic deformation process. ${ }^{31}$ Due to the reorientation in the region between twin boundaries, atoms rearrange their position collectively to accommodate the higher plastic strains. However, local configuration remains in the bcc structure and no structural phase transition occurs in either pure or oxide-coated Fe NWs during the plastic deformation process. The twin domain axial re-orientation due to the applied strain leads to local structural changes from the original [001] direction to the final [110] crystal orientation in the region between twin boundaries. The twins exhibit stochastic collective behaviors both laterally and transversely by closing and expanding the boundary by changing geometrical and crystallographic reorientation of the NW surface and the twin domain, both of which remain mobile during tensile loading. The straight twin boundaries initially move parallel to each other along the $\{112\}$ twining planes; however, at higher strain levels, the twin boundaries exhibit shift on several crystallographic as well as noncrystallographic slip planes, as is clearly shown in Figs. 7 and 8 . The resultant change of the surface and domain geometry between the twin boundaries can be attributed to simultaneous activation and operation of numerous, but partly crystallographic and non-crystallographic slip systems for all examined Fe NWs, leading to twin boundaries with irregular curved shape, as shown in Fig. 9.

The maximum critical stress is required to overcome the resistance to the initiation of partial dislocations, whereas comparatively low flow stress is sufficient for the twin boundary to propagate as strain increases, as clearly seen from the stress-strain curves. The surface oxide effect on the flow stress is less significant as, with increasing strain, both pure and oxide-coated Fe NWs showed no new twinning boundaries, whereby the existing ones tend to dominate the twinning plastic deformation. Figs. 7 and 8 present a detailed atomistic view of the initiation of partial dislocation, formation of pair of twin boundaries, and finally propagation of the twin boundary pair, which indicates that the nucleation and propagation of twinning are the major factor in the mechanical deformation mechanism for both pure and oxidecoated Fe NWs in the plastically deformed region. The twin formation and evolution path obtained by other potential models are consistent with our simulation results. Recent MD simulation studies have also revealed similar phenomena, suggesting that the onset of plastic deformation requires higher stress/energy to nucleate a twin partial dislocation on the adjacent $\{112\}$ plane, where the twin growth is relatively easier compared to the initiation in the pure Fe NW systems. ${ }^{31}$ Moreover, in our study, the observed plastic deformation mechanism in terms of initiation, propagation of the initial dislocation, formation of full stacking faults, and finally twin formation is also consistent with the MD studies of pure Fe NWs conducted by Li et al., ${ }^{31}$ who discussed
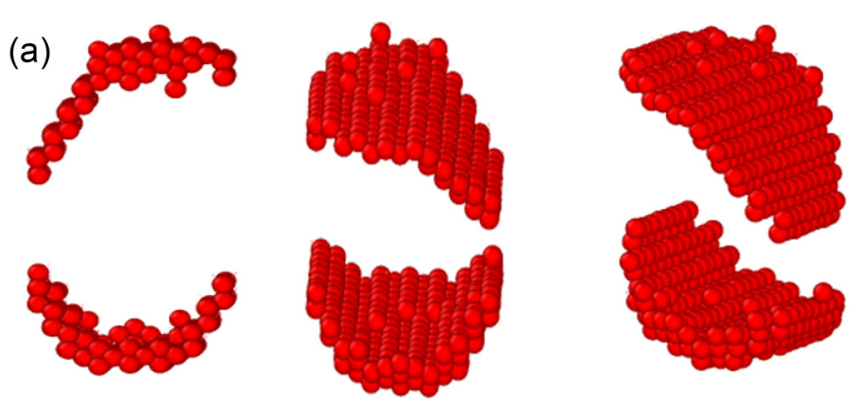

(b)
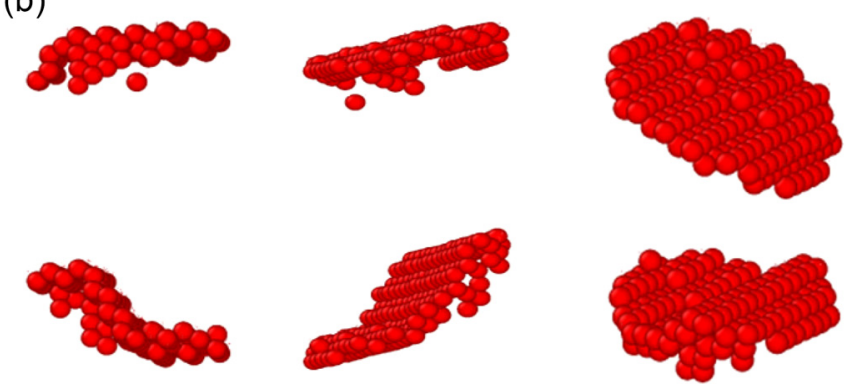

FIG. 9. To clearly observe twin boundaries, the perfect bcc and surface atoms are removed to visualize interior twin boundaries. The sequential snapshots show the twin boundaries from different perspectives for (a) the pure and (b) oxide-coated Fe NW at 16\% strain under tensile loading. Only two adjacent planes are shown in each snapshot, which become irregularly shaped at higher strain value. 
plastic and yielding behavior for metallic crystalline bcc W, Mo, W, and Fe NWs under uniaxial tension loading and unloading conditions. $\mathrm{Li}$ and colleagues pointed out that the energy barrier for emission of the first partial dislocation to form a stacking fault in a bcc metal is quite significant for twin formation, whereas a lower energy barrier for twin migration is sufficient. Extant studies on the formation of dislocations and growth of twins in metallic bec Mo, W, and Fe NWs show that the processes are similar in each case, whereby the dislocations preferentially nucleate at the $\{\overline{2} 11\}$ glide plane and all the sliding processes follow in the same mechanism. For cylindrical Fe NWs under uniaxial tensile strain in the [001] direction, initial nucleation of $\{211\}\langle 111\rangle$ dislocation glides in the $\langle 111\rangle$ direction and causes formation of the twin boundary, as reported by Byggmastar et al. ${ }^{30}$

It is generally assumed that defects, including surface oxide defects, decrease the required activation energy for the onset of plastic deformation. ${ }^{21}$ Tong et al. studied the annealing treatment effect on the structure and mechanical properties of nanocrystalline $\alpha$-iron and relevant mechanisms. ${ }^{58}$ Their results showed that the annealing treatment produced various defects, enhanced ductility, and strengthened the nanocrystalline $\alpha$-iron by reducing dislocation emission sources. The oxide defects on the Fe NW free surface drastically alter the existing homogeneity as well as symmetry of the pure Fe NWs; furthermore, the concentration of structural defects in the oxide shell layer is relatively higher than that in the pure Fe NW. ${ }^{20,21}$ Consequently, a greater number of active sites on the free surface are introduced by the oxide defects, resulting in the shift in the stress concentration to these oxide imperfections. These defect sites are more active and more sensitive to all stresses below the ideal strength, especially for metallic NWs, and increase the likelihood of emission of partial dislocations, which assist in the reduction of tensile yield stress and strain. ${ }^{50,59}$ Therefore, increasing oxide thickness layer reduces the activation barriers for the initial emission of partial dislocations, as well as overall yield strength required to conduct plastic deformation. This phenomenon has been explained in terms of various mechanisms, such as thermal strength, dislocation nucleation activation parameters, and kink formation energy. ${ }^{61,62}$ Usually, activation parameters, including the activation energy and activation volume, are used quantitatively to identify surface dislocation sources based on the kinetic rate theory. ${ }^{21,61} \mathrm{Zhu}$ et al. pointed out that the activation energy and activation volume quantitatively depend on temperature and nucleation stress, as well as NW geometry, due to the long-range elastic interaction, which is coupled with the image effects of the free sample surface. ${ }^{51,61}$ Sen and colleagues also demonstrated that the activation volume of the oxide $\mathrm{Al} \mathrm{NWs}$ for dislocation nucleation depends strongly on the nature of the oxidized interface structure ( $\mathrm{Al} /$ $\left.\mathrm{Al}_{\mathrm{x}} \mathrm{O} \mathrm{y}\right) .{ }^{21}$ In this work, the estimated dislocation nucleation activation volume for oxidized $\mathrm{Al}$ NWs was found to be $\sim 4$ times greater than that of the pure $\mathrm{Al} \mathrm{NW}$. As a result, $\mathrm{Al}$ NWs with the thinner oxide layer are more easily deformed. These results may explain why the initial defect nucleation opportunity from the oxide defect surface is enhanced, i.e., justify the observation that a thicker oxide shell layer reduces energy barrier, and increases the potential for initial partial dislocation to occur. Thus, while the complete link between the onset of plastic deformation and surface oxide defects remains elusive, their correlation can be described in terms of the kink-pair formation mechanism. To analyze quantitatively the onset of the plastic deformation process, possible explanations may be related to surface effects and the kinkpair formation energy, which is particularly relevant for the present work. The kink formation energy is calculated at $\sim 0.0256 \mathrm{eV}$ and $\sim 0.0002 \mathrm{eV}$ by using the Nose/Hoover isobaric-isothermal ensemble (NPT) under zero stress condition at room temperature for the cylindrical pure and oxidecoated Fe NWs, respectively. ${ }^{47}$ The significant difference between the kink formation energy values pertaining to pure and oxide-coated Fe NWs may probably provide a reasonable explanation for the difficulty associated with the initiation of partial dislocations on the free surface of pure Fe NW compared to the oxide-coated Fe NWs. The kink formation energy is strongly dependent on the oxide shell layer, whereby oxide defects reduce the kink-pair formation energy but increase the onset of plastic deformation mechanism. Consequently, oxide-coated Fe NWs can easily start plastic deformation on the corresponding slip plane. Using MD simulation, the kink formation energy for $1 / 2\{1 \overline{2} 1\}\langle 111\rangle$ dislocations in bulk Fe, which depends on the dislocation character and geometry, was found to be $0.15 \mathrm{eV}$ under zero stress conditions at temperatures below $300 \mathrm{~K} .^{62}$ Theoretical analyses conducted by applying kink-pair nucleation models for $\mathrm{Fe}$ indicate that slip activation occurs predominately on the $\{112\}$ planes. ${ }^{62}$ The kink formation energies for the $1 / 2\{011\}\langle 111\rangle$ and $1 / 2\{112\}\langle 111\rangle$ edge dislocations in $\mathrm{Fe}$ are estimated at $0.05 \mathrm{eV}$ and $0.04 \mathrm{eV}$ by using the MD simulations method, respectively. ${ }^{63}$

Our systematic study, combined by oxidation of $\mathrm{Fe}$ NWs, provides useful quantitative information to fully realize their latent mechanical strength under tensile loading, understand nanoscale mechanical deformation mechanisms and related properties in terms of the individual contribution of the oxide shell layer thicknesses superimposed on the cylindrical metallic Fe NWs. Our results not only confirm the importance of the surface oxide defects for controlling the mechanical properties of metallic Fe NWs but also indicate that their mechanical tensile strength can be manipulated through careful tailoring of the oxide shell layer.

\section{SUMMARY AND CONCLUDING REMARKS}

In this work, we focused on determining the dependence of native oxide layers on the complex mechanical tensile deformation process, calculating related properties, discussing plastic yielding behavior and kinetics, and capturing atomistic level details for the local structure of the oxide shell layer using the ReaxFF potential model. The effect of native oxides has been found closely linked with their mechanically induced chemical reactions, which are associated with metallic Fe corrosion and play critical roles in controlling mechanical tensile deformation mechanisms and related properties that may be tested experimentally. The surface oxide defect on the Fe NW surface enhances the 
dislocation yield from the free surface, i.e., the thicker the oxide shell layer, the higher the nucleation opportunity for initial partial dislocations. Beyond initial dislocation emission, the extended defects, such as the dislocation escape velocity, can be controlled by native surface oxide defects. In particular, the unique features of the oxide shell layers result in the reduction in the CRSS acting on the $\{112\}\langle 111\rangle$-glide plane, which affects the partial dislocation migration speed. The ductility associated with the complex twinning-dominated mechanochemical deformation mechanism is dependent on collective creation and motion of the partial dislocation process, as well as lattice and surface reorientation, which are restricted between its existing internal twin boundaries. Moreover, pure Fe NWs have a higher critical strain and stress for initial nucleation of dislocations than their oxide-coated counterparts; hence, oxide defect sites reduce the initial defect nucleation activity. As a result, the formation, growth, and propagation of twins in the pure and oxide-coated Fe NWs are qualitatively very similar irrespective of the oxide form. In other words, they require large initial maximum critical stress to nucleate the partial dislocation, followed by twinning migration that requires much lower stress. Our study has a significant practical value, as it enhances the current understanding of the mechanical tensile deformation process and related properties of the Fe NWs as a function of oxide layer thickness.

\section{SUPPLEMENTARY MATERIAL}

See supplementary material for the number density profile for oxygen and iron atoms along the radial direction (Figure 1). When the $\mathrm{O}$ atoms penetrate through the $\mathrm{Fe} \mathrm{NW}$, the $\mathrm{Fe}$ atoms migrate in the opposite direction to form and thicken the dense oxide shell layer, which causes the increase of the average oxygen number density within the developing oxide shell layer. Figure 2 shows the close perspective view of the twinning evolution in the oxide Fe NW.

\section{ACKNOWLEDGMENTS}

This work was supported by The Scientific and Technological Research Council of Turkey (TUBITAK)BIDEB 2219 through Grant No. 1059B191400364. Simulations were performed at TUBITAK ULAKBIM, High Performance and Grid Computing Center (TR-Grid eInfrastructure), and ITU National Center for High Performance Computing (UHEM).

${ }^{1}$ R. Subbaraman, S. A. Deshmukh, and S. K. R. S. Sankaranarayanan, J. Phys. Chem. C 117, 5195 (2013).

${ }^{2}$ B. Jeon, Q. V. Overmeere, A. C. T. van Duin, and S. Ramanathan, Phys. Chem. Chem. Phys. 15, 1821 (2013).

${ }^{3}$ E. Ekiert and W. Arabczyk, J. Phys. Chem. C 119, 4000 (2015).

${ }^{4}$ B. D. Chernomordik, H. B. Russell, U. Cvelbar, J. B. Jasinski, V. Kumar, T. Deutsch, and M. K. Sunkara, Nanotechnology 23, 194009 (2012).

${ }^{5}$ M. Krajewski, W. S. Lin, H. M. Lin, K. Brzozka, S. Lewinska, N. Nedelko, A. Slawska-Waniewska, J. Borysiuk, and D. Wasik, Beilstein J. Nanotechnol. 6, 1652 (2015).

${ }^{6}$ G. Sainath, B. K. Choudhary, and T. Jayakumar, Comput. Mater. Sci. 104, 76 (2015).
${ }^{7}$ H. Xiang, D. M. Jiang, J. C. Yao, Y. P. Zheng, W. Lu, G. Q. Li, H. Saito, S. Ishio, X. W. Tan, and Y. Q. Lin, J. Phys.: Conf. Ser. 266, 012022 (2011).

${ }^{8}$ G. Wang, Y. Ling, D. A. Wheeler, K. E. N. George, K. Horsley, C. Heske, J. Z. Zhang, and Y. Li, Nano Lett. 11, 3503 (2011).

${ }^{9}$ L. C. Hsu, Y. Y. Li, C. G. Lo, C. W. Huang, and G. Chern, J. Phys. D: Appl. Phys. 41, 185003 (2008).

${ }^{10}$ B. Ravel, E. E. Carpenter, and V. G. Harris, J. Appl. Phys. 91, 8195 (2002).

${ }^{11}$ Q. Han, Y. Y. Xu, Y. Y. Fu, H. Zhang, R. M. Wang, T. M. Wang, and Z. Y. Chen, Chem. Phys. Lett. 431, 100 (2006).

${ }^{12}$ H. Srivastava, P. Tiwari, A. K. Srivastava, and R. V. Nandedkar, J. Appl. Phys. 102, 054303 (2007).

${ }^{13}$ C. Wang, D. R. Baer, J. E. Amonette, M. H. Engelhard, J. Antony, and Y. Qiang, J. Am. Chem. Soc. 131, 8824 (2009).

${ }^{14}$ Y. Fu, J. Chen, and H. Zhang, Chem. Phys. Lett. 350, 491 (2001).

${ }^{15}$ T. Pan, Chem. Phys. Lett. 511, 315 (2011).

${ }^{16}$ D. A. Wheeler, G. Wang, Y. Ling, Y. Li, and J. Z. Zhang, Energy Environ. Sci. 5, 6682 (2012).

${ }^{17}$ J. Yue, X. Jiang, and A. Yu, J. Phys. Chem. C 116, 8145 (2012).

${ }^{18}$ Y. L. Chueh, M. W. Lai, J. Q. Liang, L. J. Chou, and Z. L. Wang, Adv. Funct. Mater. 16, 2243 (2006).

${ }^{19}$ A. G. Nasibulin, S. Rackauskas, H. Jiang, Y. Tian, P. R. Mudimela, S. D. Shandakov, L. I. Nasibulina, J. Sainio, and E. I. Kauppinen, Nano Res. 2, 373 (2009).

${ }^{20}$ F. G. Sen, Y. Qi, A. C. T. van Duin, and A. T. Alpas, Appl. Phys. Lett. 102, 051912 (2013).

${ }^{21}$ F. G. Sen, A. T. Alpas, A. C. T. van Duin, and Y. Qi, Nat. Commun. 5, 3959 (2014).

${ }^{22}$ C. C. Rohlig, M. Niebelschutz, K. Brueckner, K. Tonisch, O. Ambacher, and V. Cimalla, Phys. Status Solidi B 247, 2557 (2010).

${ }^{23}$ Z. L. Wang, Z. R. Dai, R. P. Gao, Z. G. Bai, and J. L. Gole, Appl. Phys. Lett. 77, 3349 (2000).

${ }^{24}$ M. D. Skarlin and D. J. Quesnel, J. Appl. Phys. 118, 235306 (2015).

${ }^{25}$ P. Landau, Q. Guo, P. Hosemann, Y. Wang, and J. R. Greer, Mater. Sci. Eng., A 612, 316 (2014).

${ }^{26}$ G. Sainath, B. K. Choudhary, and T. Jayakumar, "Deformation behaviour of body centered cubic $\mathrm{Fe}$ nanowires under tensile and compressive loading," preprint arXiv:1409.3324 (2014).

${ }^{27}$ G. Sainath and B. K. Choudhary, Comput. Mater. Sci. 111, 406 (2016).

${ }^{28}$ C. J. Healy and G. J. Ackland, Acta Mater. 70, 105 (2014).

${ }^{29}$ D. J. Hepburn and G. J. Ackland, Phys. Rev. B 78, 165115 (2008).

${ }^{30}$ J. Byggmästar, F. Granberg, A. Kuronen, K. Nordlund, and K. O. E. Henriksson, J. Appl. Phys. 117, 014313 (2015).

${ }^{31} \mathrm{~S}$. Li, X. Ding, J. Deng, T. Lookman, J. Li, X. Ren, J. Sun, and A. Saxena, Phys. Rev. B 82, 205435 (2010).

${ }^{32}$ J. Wang, Z. Zeng, C. R. Weinberger, Z. Zhang, T. Zhu, and S. X. Mao, Nat. Mater. 14, 594 (2015).

${ }^{33}$ M. Aryanpour, A. C. T. van Duin, and J. D. Kubicki, J. Phys. Chem. A 114, 6298 (2010).

${ }^{34}$ T. J. Campbell, G. Aral, S. Ogata, R. K. Kalia, A. Nakano, and P. Vashishta, Phys. Rev. B 71, 205413 (2005).

${ }^{35}$ A. Perron, S. Garruchet, O. Politano, G. Aral, and V. Vignal, J. Phys. Chem. Solids 71, 119 (2010).

${ }^{36}$ A. Hasnaoui, O. Politano, J. M. Salazar, and G. Aral, Phys. Rev. B 73, 035427 (2006).

${ }^{37}$ X. W. Zhou and H. N. G. Wadley, J. Phys.: Condens. Matter 17, 3619 (2005).

${ }^{38}$ F. H. Streitz and J. W. Mintmire, Phys. Rev. B 50, 11996 (1994).

${ }^{39}$ K. I. Nomuro, R. K. Kalia, A. Nakano, and P. Vashishta, Comput. Phys. Commun. 178, 73 (2008).

${ }^{40}$ S. Plimpton, J. Comput. Phys. 117, 1 (1995).

${ }^{41}$ U. Khalilov, G. Pourtois, A. C. T. van Duin, and E. C. Neyts, Chem. Mater. 24, 2141 (2012).

${ }^{42}$ O. Assowe, O. Politano, V. Vignal, P. Arnoux, B. Diawara, O. Verners, and A. C. T. van Duin, J. Phys. Chem. A 116, 11796 (2012).

${ }^{43}$ S. Y. Kim, N. Kumar, P. Persson, J. Sofo, A. C. T. van Duin, and J. D. Kubicki, Langmuir 29, 7838 (2013).

${ }^{44}$ D. Fantauzzi, J. Bandlow, L. Sabo, J. E. Mueller, A. C. van Duin, and T. Jacob, Phys. Chem. Chem. Phys. 16, 23118 (2014).

${ }^{45}$ W. J. Mortier, S. K. Ghosh, and S. Shankar, J. Am. Chem. Soc. 108, 4315 (1986).

${ }^{46}$ G. J. Martyna, M. L. Klein, and M. Tuckerman, J. Chem. Phys. 97, 2635 (1992). 
${ }^{47}$ G. J. Martyna, D. J. Tobias, and M. L. Klein, J. Chem. Phys. 101, 4177 (1994).

${ }^{48}$ M. P. Allen and L. J. Tildesley, Computer Simulation of Liquids (Oxford University Press, New York, 1987).

${ }^{49}$ L. Yuan, Y. Wang, R. Cai, Q. Jian, J. Wang, B. Li, A. Sharma, and G. Zhou, Mater. Sci. Eng., B 177, 327 (2012).

${ }^{50}$ D. M. Clatterbuck, D. C. Chrzan, and J. W. Morris, Acta Mater. 51, 2271 (2003).

${ }^{51}$ T. Zhu, J. Li, A. Samanta, A. Leach, and K. Gall, Phys. Rev. Lett. 100, 025502 (2008).

${ }^{52}$ B. T. H. L. Khanh, V. V. Hoang, and H. Zung, Eur. Phys. J. D 49, 325 (2008).

${ }^{53}$ W. Zhonghua, G. Lin, L. Qianshu, and Z. Hesun, J. Phys.: Condens. Matter 11, 4961 (1999).
${ }^{54}$ Y. Zhang, D. J. Yu, and K. M. Wang, J. Mater. Sci. Technol. 28, 164 (2012).

${ }^{55}$ L. Y. Lin, Z. Ying, H. R. Jie, and L. G. Hong, Chin. Phys. B 18, 1923 (2009).

${ }^{56}$ R. A. Kellogg, A. M. Russell, T. A. Lograsso, A. B. Flatau, A. E. Clark, and M. Wun-Fogle, Proc. SPIE 5053, 534 (2003).

${ }^{57}$ J. J. Adams, D. S. Agosta, R. G. Leisure, and H. Ledbetter, J. Appl. Phys. 100, 113530 (2006).

${ }^{58}$ X. Tong, H. Zhang, and D. Y. Li, Sci. Rep. 5, 8459 (2015).

${ }^{59}$ A. Stukowski, Modell. Simul. Mater. Sci. Eng. 18, 015012 (2010).

${ }^{60}$ B. R. S. Rogne and C. Thaulow, Philos. Mag. 95, 1814 (2015).

${ }^{61}$ T. Zhu and J. Li, Prog. Mater. Sci. 55, 710 (2010).

${ }^{62}$ T. D. Swinburne, S. L. Dudarev, S. P. Fitzgerald, M. R. Gilbert, and A. P. Sutton, Phys. Rev. B 87, 064108 (2013).

${ }^{63}$ C. L. Qun, W. C. Yu, and Y. Tao, Chin. Phys. B 17, 662 (2008). 